\title{
Breakage and coalescence of drops in turbulent stirred dispersions
}

\author{
D K R NAMBIAR, R KUMAR* and K S GANDHI \\ Department of Chemical Engineering, *and also Jawaharlal Nehru Centre \\ for Advanced Scientific Research, Indian Institute of Science, Bangalore \\ 560012 , India
}

MS received 4 September 1990

\begin{abstract}
The various existing models for predicting the maximum stable drop diameter $d_{\max }$ in turbulent stirred dispersions have been reviewed. Variations in the basic framework dictated by additional complexities such as the presence of drag reducing agents in the continuous phase, or viscoelasticity of the dispersed phase have been outlined. Drop breakage in the presence of surfactants in the continuous phase has also been analysed. Finally, the various approaches to obtaining expressions for the breakage and coalescence frequencies, needed to solve the population balance equation for the number density function of the dispersed phase droplets, have been discussed.
\end{abstract}

Keywords. Drop breakage; coalescence of drops; stirred vessels; size distribution of drops in stirred vessels; turbulence; maximum stable drop diameter; breakage frequency; coalescence frequency.

\section{Introduction}

Mechanical agitation is a commonly employed method for generating and maintaining a dispersion of one liquid phase in another. Two approaches are available to quantitatively predict the rates of heat and mass transport in such dispersions. The commonly used approach considers all the drops existing in the vessel to be identical in size, and as having identical properties. Thus, an average drop size, an average transfer coefficient and a uniform driving force are used. The average drop size, called the 'Sauter mean diameter', $d_{32}$, is defined in such a way that the total surface area available for transport is conserved. The area per unit volume of the dispersion can then be calculated by

$$
a=6 \phi / d_{32} \text {. }
$$

As $d_{32}$ does not have a theoretical basis which can be exploited for its prediction,

A list of symbols is given at the end of the paper 
attempts have been made to predict $d_{\max }$, the diameter of the largest drop that cannot be further broken in the vicinity of the impeller. The value of $d_{32}$ is then determined from $d_{\max }$ by dividing it with an empirically obtained factor (lying between 1.5 and 1.6 for most systems). The main difficulty with the averaging method is that it bypasses the fact that most transport processes can vary widely from drop to drop. Schumpe \& Deckwer (1980) have shown that averaging can lead to results significantly different from those obtained experimentally. Further, the mixing of the dispersed phase depends on coalescence and breakage of drops which occur at finite rates, whereas the averaging technique assumes infinite rates of coalescence and breakage. A prelude to such work that accounts for these processes must be the description of just the coalescence and breakage processes. Thus attempts are being made to consider individual drop sizes along with their rates of coalescence and breakage to obtain a more realistic description of a stirred dispersion. This is achieved through the frame work of population balance equations (Hulburt \& Katz 1964).

The number balance equation for the dispersed phase droplets can be written as:

$$
\begin{aligned}
& \frac{\partial n(v, t)}{\partial t}=\int_{v}^{\infty} \Gamma\left(v^{\prime}\right) n\left(v^{\prime}, t\right) \beta\left(v, v^{\prime}\right) \gamma\left(v^{\prime}\right) \mathrm{d} v^{\prime}- \\
&-\Gamma(v) n(v, t)+\int_{0}^{v / 2} q\left(v-v^{\prime}, v^{\prime}\right) n\left(v-v^{\prime}, t\right) n\left(v^{\prime}, t\right) \mathrm{d} v^{\prime}- \\
&-\int_{0}^{\infty} q\left(v, v^{\prime}\right) n(v, t) n\left(v^{\prime}, t\right) \mathrm{d} v^{\prime}+N_{0}(v, t)-n(v, t) f_{0}(v) .
\end{aligned}
$$

The first term on the right hand side of the above equation represents the rate of formation of drops of size $v$ due to breakage of drops of size larger than $v$. The second term denotes the rate of disappearance of drops of volume $v$ due to breakage. The third term signifies the rate of formation of drops of size $v$ due to coalescence between droplets of volume $v-v^{\prime}$ and $v^{\prime}$. The fourth term describes the rate at which drops of volume $v$ are lost due to their coalescence with other drops. The fifth and sixth terms denote the input and escape rates of the drops of volume $v$. The left hand side of the equation is the rate of accumulation of drops of volume $v$, as a result of their birth and death due to breakage and coalescence, as well as feed and escape.

The fifth and sixth terms of (2) vanish for a batch system. In order to solve the population balance equation, a knowledge of the breakage frequency, the probability distribution of the size of the daughter droplets, the number of fragments formed upon breakage, and the coalescence frequency are needed. Separate models are required to obtain expressions for various terms involving breakage and coalescence of drops, which are then incorporated in the population balance equation.

A brief outline of the paper is given below. As the knowledge of $d_{\max }$ is important for the evaluation of both $d_{32}$ and breakage frequency, the basic framework required for its prediction is first discussed along with the information about turbulence in stirred vessels. Based on this framework, models for predicting $d_{\max }$ for rheologically complex dispersed phases and for situations where surfactants or drag reducing agents are added to the continuous phase are then discussed. All these models assume that a drop invariably breaks into two equal drops. It is then shown that the drops can break into two unequal parts. The conditions under which this could happen are then modelled. The breakage frequency models based on equal and unequal breakage 
follow in natural sequence. It is shown that the unequal breakage model, along with the energy cascade theory of distribution of energy into eddies of different length scales, can predict not only the breakage frequency but also the drop size distribution of the daughter droplets. The drop size distribution for a breakage controlled situation is then obtained by using a simplified population balance equation. Coalescence frequency is the product of collision frequency and coalescence efficiency. Models for both these are described. At the end of each major section, a short discussion is presented regarding the weaknesses in our present understanding and the problems to be solved by further work.

\section{Drop breakage in stirred vessels}

Understanding of the breakage phenomenon is necessary both for the prediction of $d_{\max }$ (which in turn is used for determining $d_{32}$ ) and for obtaining expressions for breakage related terms like breakage frequency to be used in the population balance equations.

Most of the models available up to this time assume that breakage primarily occurs because of the pressure difference operating across the drop due to turbulent velocity fluctuations. The drop experiencing these stresses tends to deform away from the spherical shape and hence experiences a shape restoring stress due to interfacial tension. Further, if the flow within the drop is assumed to be laminar, there will be a viscous stress retarding the rate of deformation. The deformation of the drop is three-dimensional. Unfortunately we do not yet have a clear picture of the threedimensional flow field in the vicinity of the drop. Hence one-dimensional models, which capture the gross features of the breakage phenomenon but bypass the detailed flow field, are being developed at this stage.

\subsection{Turbulence characteristics of stirred vessels}

A stirred vessel along with the Rushton impeller and baffles is shown in figure 1. Different types of impellers are employed in stirred vessels, depending on the flow characteristics desired. The Rushton turbine impeller is normally used for dispersions, whereas other types of impellers are employed when high circulation rates are desired. Several workers (Nishikawa et al 1976; Okamoto et al 1981; Benyad et al 1985; Bujalski et al 1987; Costes \& Coudrec 1988a, b) have reported data on turbulence intensities, autocorrelation functions, turbulence scales, energy spectra, and turbulence energy dissipation rates in stirred vessels. In general it is found that $60 \%$ of the energy transmitted to the liquid by the impeller is dissipated in the region around the impeller; the volume of this region being only about $10 \%$ of the total volume. Thus the energy dissipation rates in this zone are nearly fifteen times as large as that in the region away from the impeller. It is observed that the energy spectra show a $-5 / 3$ slope in the higher frequency range and this has been the basis for assuming that the turbulence in the impeller zone is homogeneous and isotropic. This facilitates the application of the energy cascade theory to estimate the energy contained in the eddies in the inertial subrange, the size range occupied by the drops produced in the stirred vessels.

The above picture however shows that hydrodynamics in a stirred vessel is quite complex with zones of high turbulence intensity as well as zones of low turbulence 

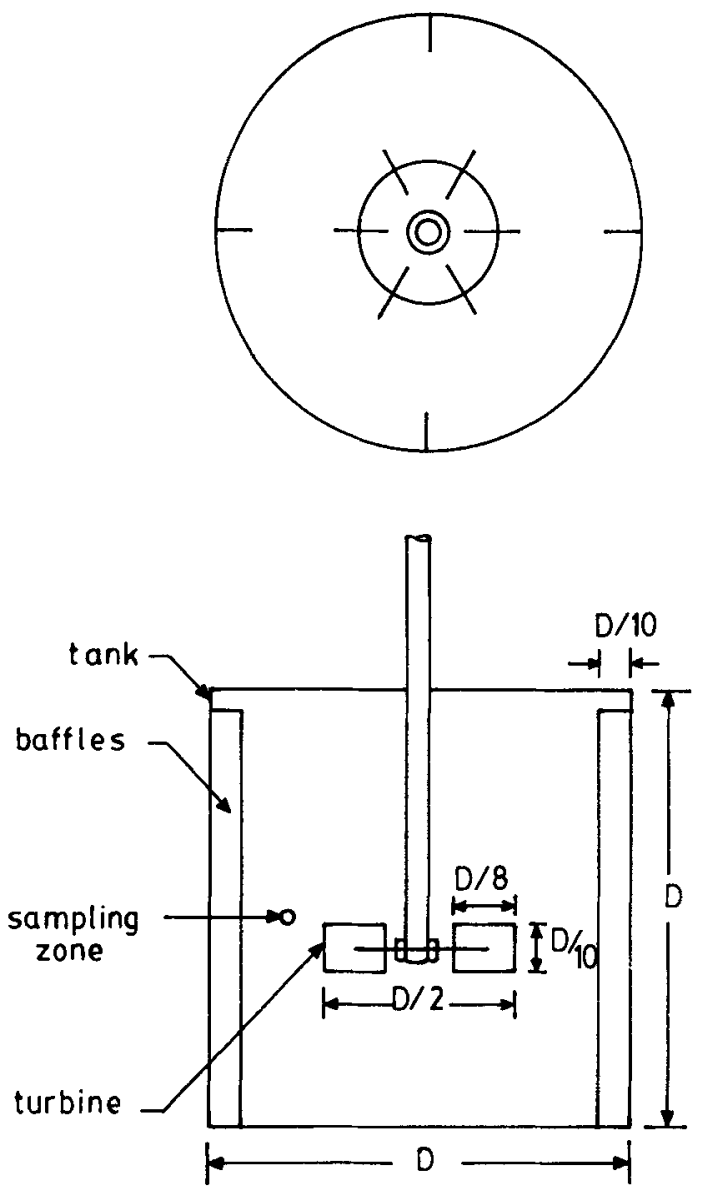

Figure 1. The stirred vessel.

intensity where viscous shear could be more important. This could be of relevance to both breakage as well as coalescence phenomena since these depend crucially on the type of flow field in which they take place.

The above reported measurements are in homogeneous media. A dispersion is however a heterogeneous system and as the volume occupied by the dispersed phase increases, the turbulence characteristics are bound to be altered. Due to the experimental difficulties involved, measurements on such systems have so far not been reported and this imposes serious limitations on theoretical developments in dispersions with high volume fractions.

For all the analyses available, it is assumed that the isotropic turbulence theory can be used. This is justified to some extent as breakage is likely to occur in the zone surrounding the impeller where the turbulence intensity is very high. Experimental measurements also show that the drop size increases as we move farther away from the impeller.

Turbulence has been associated with the presence of eddies, often visualized as simultaneously rotating and elongating fluid elements. The application of turbulent stress on the drop is referred to as drop-eddy interaction, which lasts till the lifetime of the eddy. The largest eddy present in the vessel cannot be larger than the blade size of the impeller. The eddy size decreases thereby resulting in an energy cascade. 
Eventually the energy of the eddies gets dissipated as heat when the eddy sizes reach the Kolmogorov scale. As the eddy size decreases, the energy associated with the eddy as well as its lifetime decrease. If a drop interacts with a much larger eddy it simply gets convected. As the eddy size becomes smaller and nearly equal to the drop size it is able to deform the drop. As both the lifetime and energy of a large eddy are high, and the restoring stress for a big drop is small, the large eddy is able to break a drop of equal size. With decreasing drop and eddy sizes, the lifetime and energy of the eddy will decrease whereas the shape-restoring interfacial tension stress will increase. A drop size is finally reached which cannot be broken by the eddy in its lifetime. This drop size is the maximum possible one existing near the impeller and is referred to as $d_{\max }$. This conceptual picture can form the basis of predictive theories for $d_{\max }$. It is assumed in all these models that the same stress remains on a drop for the lifetime of the eddy in spite of the fact that the drop is moving.

It has been found that the Sauter mean diameter is approximately $60 \%$ of $d_{\max }$. Although this relationship can be wrong by about $30 \%$ in case of very viscous dispersed phases, the conceptual convenience with which theories can be developed for $d_{\max }$ has led several investigators to focus on predicting $d_{\max }$.

\subsection{Models for the prediction of $d_{\max }$}

2.2a Dispersed phases of low viscosity: Kolmogorov (1949) and Hinze (1955) were the very first to develop expressions for $d_{\max }$. The kinetic energy of eddies of size $d$ is proportional to $\rho_{\mathrm{c}} \overline{u^{2}(d)} d^{3}$ while the surface energy of a drop of size $d$ is proportional to $\sigma d^{2}$. The two energies are just equal to each other when $d$ is equal to $d_{\max }$, if viscous resistance inside the drop is negligible. Thus,

$$
\rho_{c} \overline{u^{2}\left(d_{\max }\right)} d_{\max }^{3}=k_{1} \sigma d_{\max }^{2}
$$

The mean of square of velocity fluctuations in an eddy in the inertial subrange in an isotropic turbulent flow field is given by

$$
\overline{u^{2}(d)} \propto \varepsilon^{2 / 3} d^{2 / 3}
$$

Further in a stirred vessel, it is usually found that

$$
\varepsilon \propto N^{3} D^{2} \text {. }
$$

Substitution of (4) and (5) into (3) yields,

$$
d_{\max } / D=C^{\prime} \mathrm{We}^{-0 \cdot 6} \text {. }
$$

Equation (6) is a classical equation that has been used for lean dispersions of inviscid liquids for many years. Sprow (1967) has found the constant $C^{\prime}$ to vary between $0 \cdot 126$ and $0 \cdot 15$. Lagisetty et al (1986) found this constant to be $0 \cdot 125$. Coulaloglou \& Tavlarides (1976) have discussed the various correlations, based on this equation, available in the literature.

$2.2 \mathrm{~b}$ Viscous and rheologically complex dispersed phases: It has been reported by many workers (Arai et al 1977; Konno et al 1982; Lagisetty et al 1986; Calabrese et al 1986; Davies 1987) that the maximum drop size increases with increase in the dispersed phase viscosity. Equation (6) cannot be used to predict the effect of dispersed 
phase viscosity as it neglects the viscous forces generated in a drop prior to its breakage. Arai et al (1977) were the first to propose a model for predicting $d_{\max }$ which incorporated the effect of dispersed phase viscosity. They described the breakage phenomenon through a Voigt element which simultaneously took into account the restoring stress due to interfacial tension as well as the viscous stress due to flow inside the drop. They assumed that the turbulent pressure fluctuation is periodic and that the drop breaks when the deformation strain reaches a critical value. They obtained a semi-empirical correlation for $d_{\max }$ in terms of Weber number and a grouping that includes viscosity of the dispersed phase. Lagisetty et al (1986) have pointed out that the Voigt model, as has been applied, has a maximum equilibrium deformation and is reversible. They also felt that the periodic pattern assigned to the turbulent stress is not realistic. Moreover, the model did not give the low viscosity limit naturally and hence this limit had to be introduced in an ad hoc manner. They considered that the basic phenomenon could indeed be expressed by a Voigt model, but one that is modified to overcome the deficiencies of the models of Konno et al (1982) and Arai et al (1977). They assumed that with the increase in deformation, the restoring elastic stresses due to interfacial tension first increase, pass through a maximum and then decrease. The depicted role of interfacial tension is in the right direction as Rallison (1984) has observed in the context of drop break-up in shear flows. As the drop approaches the break-up condition, interfacial tension actually aids the fragmentation process. Lagisetty et al (1986) assumed that the force due to interfacial tension reaches zero when the dimensionless deformation of the drop $\theta$ becomes unity. They also assumed that the drop break-up process should be completed within the lifetime of the eddy. Thus, for breakage to occur, the value of $\theta$ should reach unity within the lifetime of the eddy. Their model has been schematically shown in figure 2. The basic equations for their model are:

$$
\begin{array}{rlr}
\tau_{t} & =\tau_{s}+\tau_{v}, & \\
\tau_{s} & =(\sigma / d) \theta,\left(1-\theta_{s}\right), \quad \theta_{s} \leqslant 1 \\
& =0, & \theta_{s} \geqslant 1 \\
\tau_{v} & =\tau_{0}+K\left(\mathrm{~d} \theta_{v} / \mathrm{d} t\right)^{n} .
\end{array}
$$

Equation (9) is the general constitutive equation yielding Newtonian, power law and Bingham plastic fluids as special cases. The average turbulent stress in a stirred vessel across a length scale of $d$ is proportional to $\rho_{c} \overline{u^{2}(d)}$ and can be expressed as

$$
\tau_{t}=C \rho_{c} N^{2} D^{4 / 3} d^{2 / 3}
$$

As the Voigt model's elements act in parallel,

$$
\theta_{v}=\theta_{s}=\theta
$$

Substituting (8), (9), (10) and (11) into (7), we obtain

$$
C \rho_{c} N^{2} D^{4 / 3} d^{2 / 3}-\tau_{0}=(\sigma / d) \theta(1-\theta)+K(\mathrm{~d} \theta / \mathrm{d} t)^{n} .
$$

Equation (11) may be expressed in dimensionless form as:

$$
C \mathrm{We}(d / D)^{5 / 3}-\left(\theta-\theta^{2}\right)-\left(\tau_{0} d / \sigma\right)=(\mathrm{d} \theta / \mathrm{d} \eta)^{n},
$$




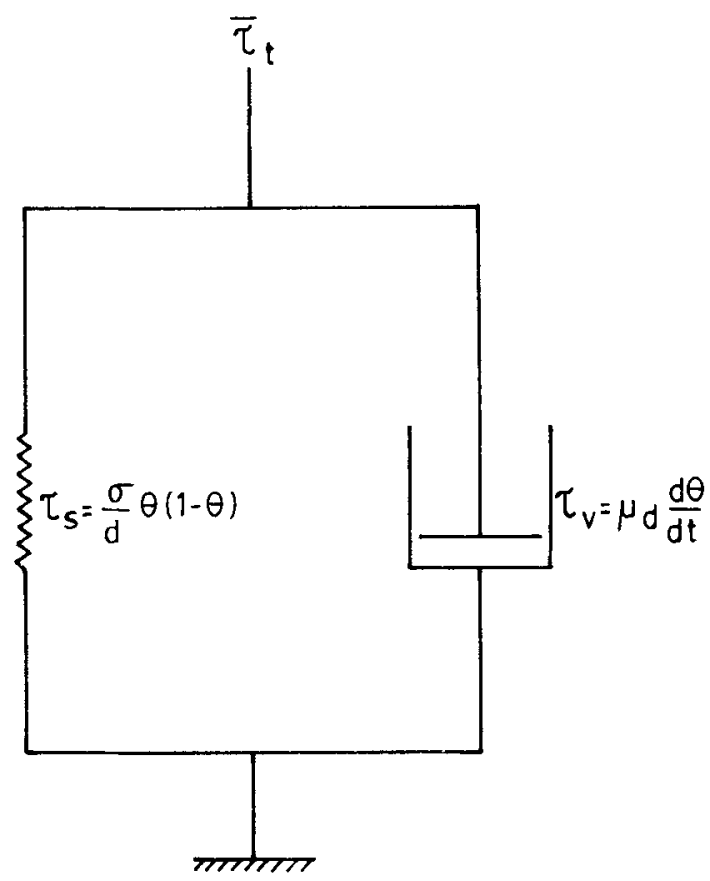

Figure 2. A Voigt element.

where $\eta$ is the nondimensional time given by $t /(K d / \sigma)^{1 / n}$. The initial condition for this equation is given by:

$$
\theta=1, \text { at } \eta=0 .
$$

Equation (14) is solved to find $\eta$ at $\theta=1$. If $\eta$ at $\theta=1$ is more than the nondimensional lifetime of the eddy, breakage would not occur. Thus, for breakage to occur, the following condition must be satisfied:

$$
\eta(\theta=1) \leqslant \bar{T} /(K d / \sigma)^{1 / n} .
$$

The maximum diameter of the stable drop is that for which the following equality holds:

$$
\eta(\theta=1)=\bar{T} /\left(K d_{\max } / \sigma\right)^{1 / n}
$$

The $d_{\max }$ can therefore be determined by solving (13), (14) and (16) provided an expression is available for $\bar{T}$. Using the turnover time of the eddy as the lifetime of the eddy:

$$
\bar{T}=(1 / N)(d / D)^{2 / 3} .
$$

Lagisetty et al (1986) have given solutions for various values of $n$, including the value of unity. They found that the value of $C$ works out to be equal to 8 . Their model gives (6) as a limit as $K \rightarrow 0$ and, with $C=8$, the proportionality constant equals 0.125 , a value close to that reported by Sprow (1967). The solution for Newtonian fluids is given by

$$
\left(\operatorname{Re}_{/} / \mathrm{We}\right)\left(d_{\max } / D\right)^{-1 / 3}=\left[1 /(4 \chi-1)^{\frac{1}{2}}\right] \tan ^{-1}\left[1 /(4 \chi-1)^{\frac{1}{2}}\right],
$$




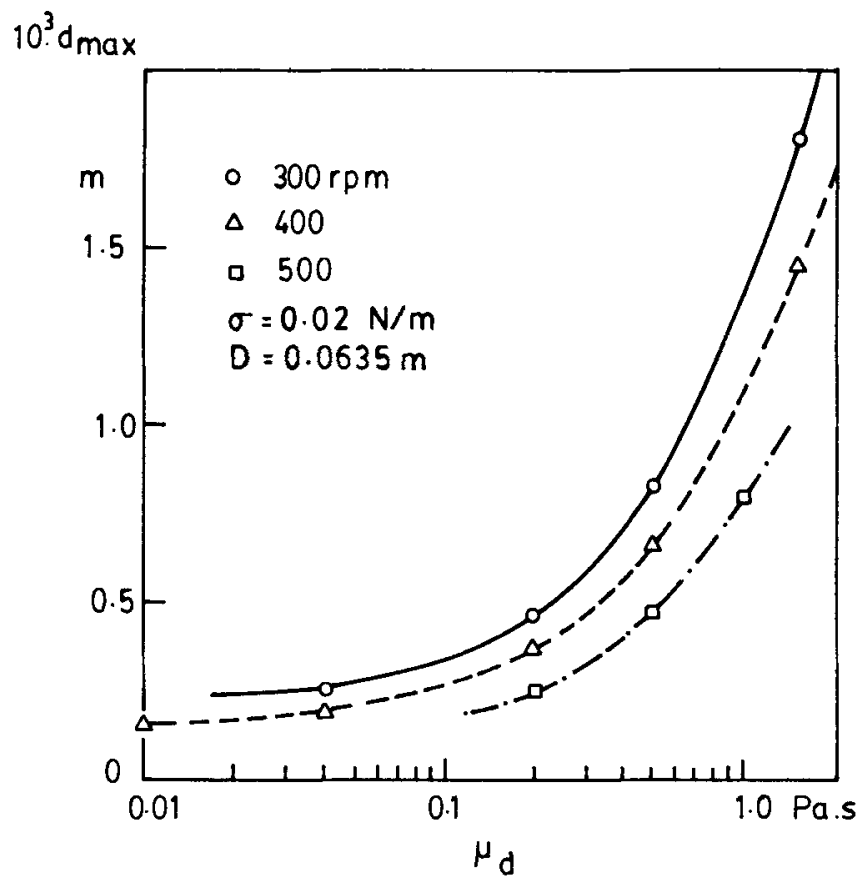

Figure 3. Verification of the model of Lagisetty et al (1986) for the effect of dispersed phase viscosity $\left(\mu_{d}\right)$ on $d_{\max }$. The lines correspond to the theory (18) and are compared with the experimental results of Arai et al (1977).

where $\chi$ is equal to $8 \mathrm{We}\left(d_{\max } / D\right)^{5 / 3}$. Figure 3 shows a comparison between theoretical predictions and experimental observations for the effect of dispersed phase viscosity on $d_{\max }$. It can be seen that, when interfacial tension is of the order of $0.02 \mathrm{~N} / \mathrm{m}, d_{\max }$ is hardly affected by the dispersed phase viscosity till it exceeds about $0 \cdot 1$ Pa.s. They have tested the model using Newtonian, power law and Bingham plastic dispersed phases and report the agreement between the predicted and experimentally obtained values to be good. Some recent measurements on very viscous liquids however seem to indicate that Lagisetty's model overpredicts the value of $d_{\max }$ under these extreme conditions.

The basic framework of the model has been tested for a number of situations where either surfactants (or drag reducing agents) were added to the continuous phase, or the dispersed phase was a rheologically more complex viscoelastic fluid.

2.2c Breakage in presence of surfactants: Koshy et al (1988a) have investigated the breakage of dispersed phases when a surfactant is added to the continuous phase. Addition of surfactant could be expected to reduce the drop diameter by reducing the interfacial tension of the system. Two systems with identical interfacial tensions, one with surfactant and the other without it, were studied. The water-octanol system had an interfacial tension of $0.0083 \mathrm{~N} / \mathrm{m}$ and did not have a surfactant. Teepol was added to a water-styrene system to bring its interfacial tension from $0.034 \mathrm{~N} / \mathrm{m}$ to $0.0083 \mathrm{~N} / \mathrm{m}$. The viscosities of both the dispersed phases were too low to affect $d_{\max }$ and in any case were nearly equal. The values of $d_{\max }$ have been plotted as a function of revolutions per second (rps) for both the systems in figure 4. It is interesting to note that the two systems yield different results and, surprisingly, while (6) explains 


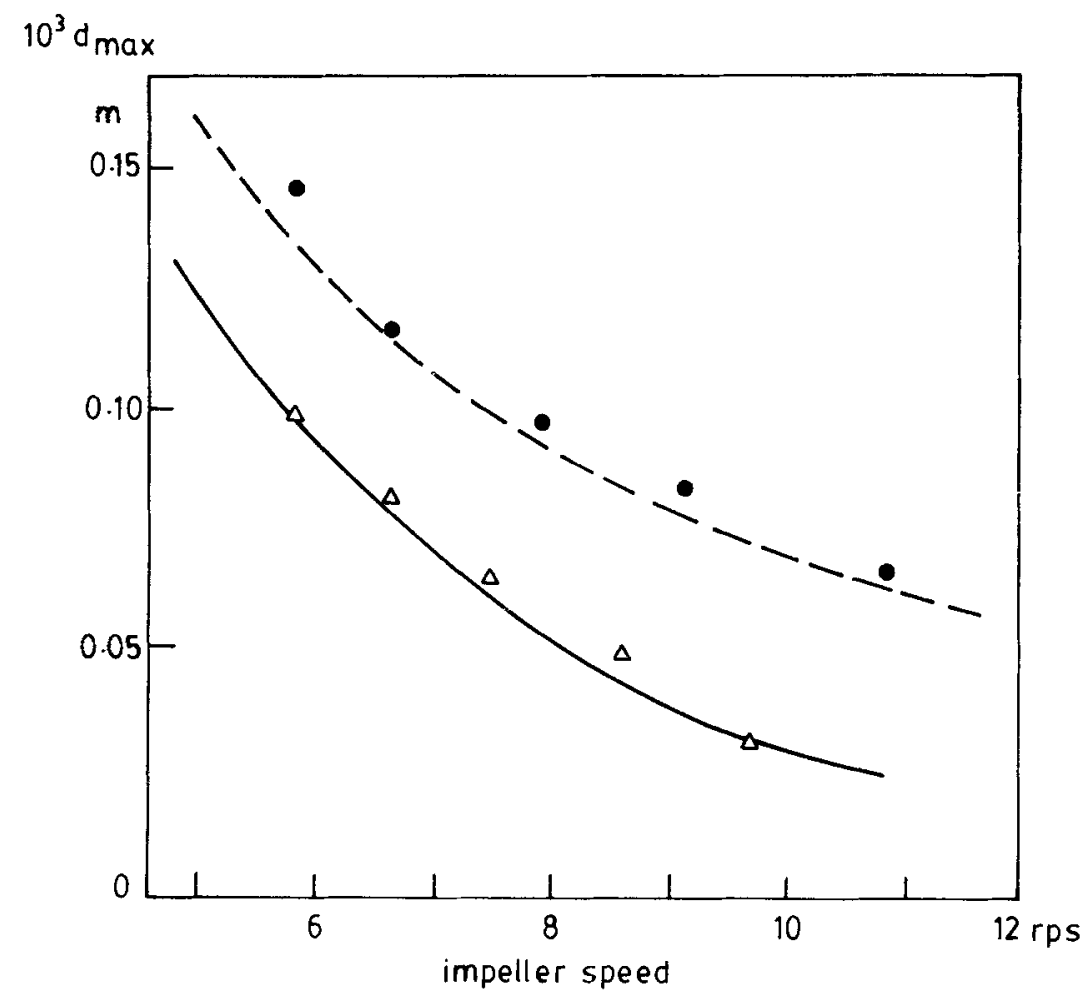

Figure 4. Breakage in presence of surfactants. The experimental values for the water-octanol system $(-)$ are in good agreement with $(6)(-\ldots)$. The best fit line $(-\longrightarrow$ through the experimental data $(\Delta)$ for a surfactant solution-styrene system having the same interfacial tension $(0.0083 \mathrm{~N} / \mathrm{m})$ indicate lower $d_{\max }$ values in the presence of a surfactant.

the results of the water-octanol system, using a value of 0.125 for the constant, it fails to explain the results of the system containing the surfactant. These results indicate that the surfactant not only acts through reduction of the interfacial tension, but also influences the breakage in some other fashion which has not been considered by any of the earlier models.

A pressure fluctuation across a drop diameter most probably causes a depression or elongation which propagates, resulting in drop breakage. When surfactants are present at the interface, the pressure fluctuation, apart from causing depression at the surface, also removes the adsorbed surfactant molecules thereby exposing fresh interface. This fresh interface has dynamic interfacial tension, which is higher than the static interfacial tension. This difference in the two interfacial tensions causes a flow towards the base which adds to the flow already taking place due to pressure fluctuation. The assumed mechanism through which the extra stress is generated has been shown in figure 5. The model of Lagisetty et al (1986) has been modified to account for this extra stress as follows:

$$
C \rho_{\mathrm{c}} N^{2} D^{4 / 3} d^{2 / 3}+(\Delta \sigma / d)-\tau_{0}=(\sigma / d) \theta(1-\theta)+K(\mathrm{~d} \theta / \mathrm{d} t)^{n}
$$

where $\Delta \sigma$ is the difference between the dynamic and static interfacial tension values. The modified model has been tested by Koshy et al (1988a). Figure 6 shows two sets of experimental data in the range of surfactant concentrations where the dynamic 

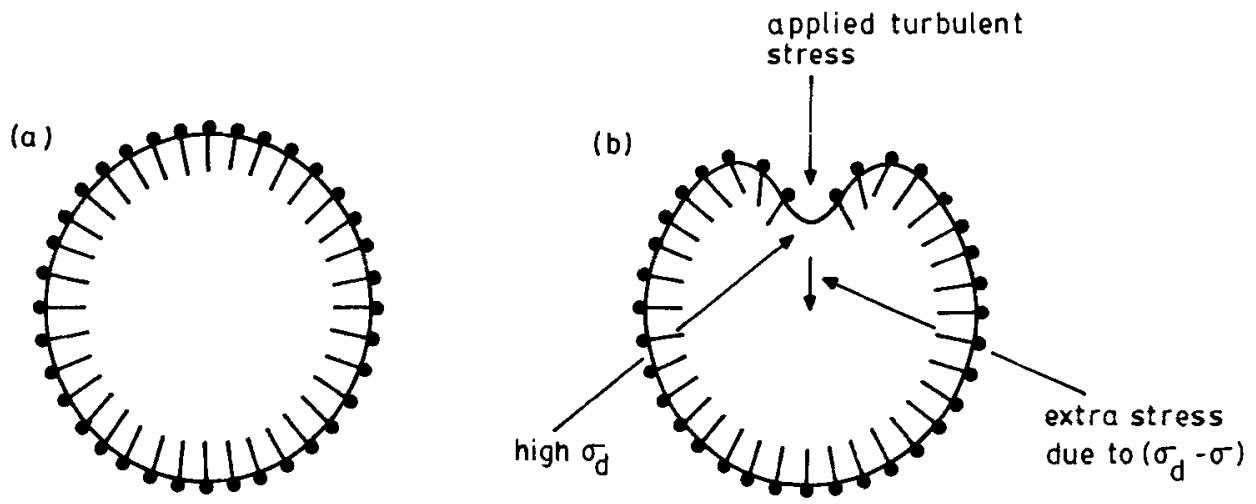

Figure 5. Mechanism through which a surfactant influences drop breakage. (a) Drop before interation with eddy; (b) formation of the depression.

and equilibrium interfacial tensions differ. For both sets of data presented, the system is water-styrene. Different concentrations of Teepol have been used for the two sets. In one of the sets, the interfacial tension has been reduced to $0.011 \mathrm{~N} / \mathrm{m}$ while the $\Delta \sigma$ is $0.0014 \mathrm{~N} / \mathrm{m}$. The points are experimental whereas the solid and dotted lines correspond to (19) and to Lagisetty et al's (1986) model respectively. It is evident that the model represented by (19) is able to predict the results correctly while the other overpredicts the $d_{\max }$ values significantly. Similar conclusions can be drawn from the other set, the interfacial tension and $\Delta \sigma$ for which are 0.0253 and $0.0011 \mathrm{~N} / \mathrm{m}$ respectively.

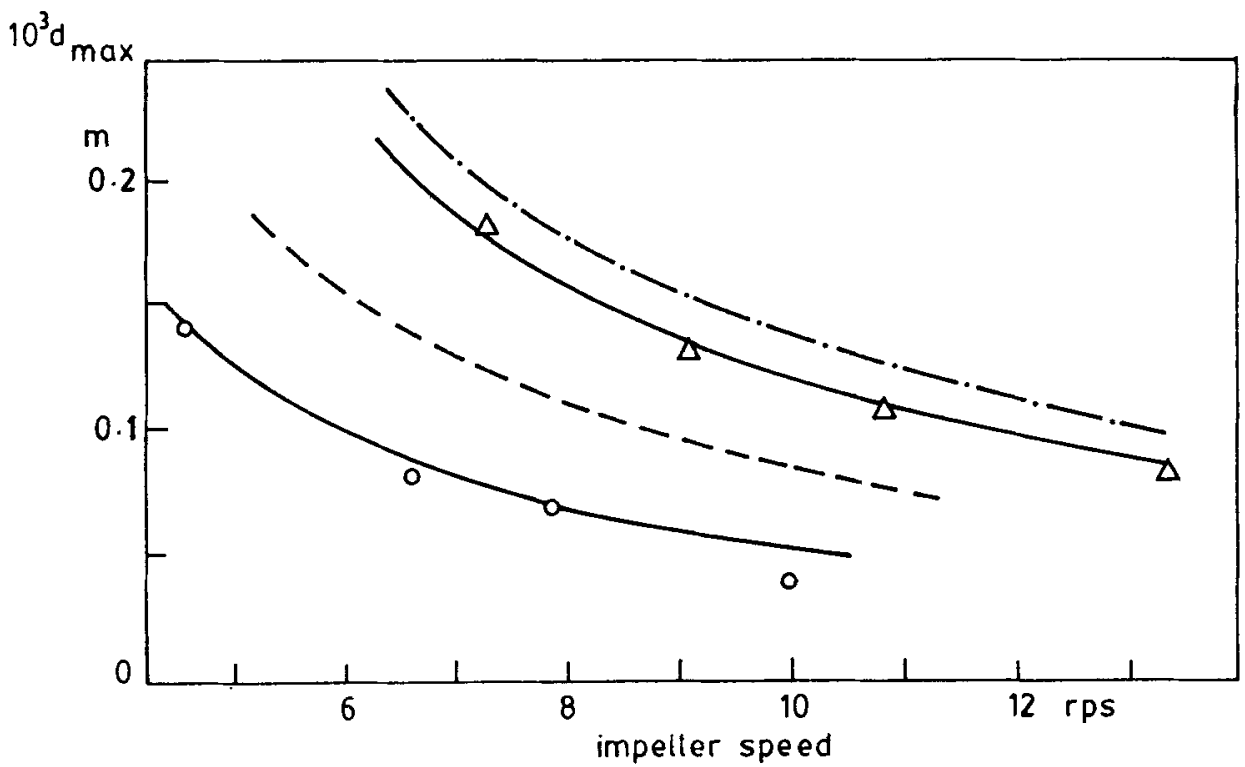

Figure 6. Effect of surfactant on $d_{\max }$ when the dynamic and static interfacial tensions are different. The system is styrene-water and the surfactant used is Teepol. Two different concentrations have been employed $-(O): \sigma=0.011 \mathrm{~N} / \mathrm{m} ; \Delta \sigma=0.0014 \mathrm{~N} / \mathrm{m}$ and $(\Delta)$ : $\sigma=0.0253 \mathrm{~N} / \mathrm{m} ; \Delta \sigma=0.0011 \mathrm{~N} / \mathrm{m}$. The dashed lines represent (18) while the solid lines correspond to the model of Koshy et al 1988a, (19). 


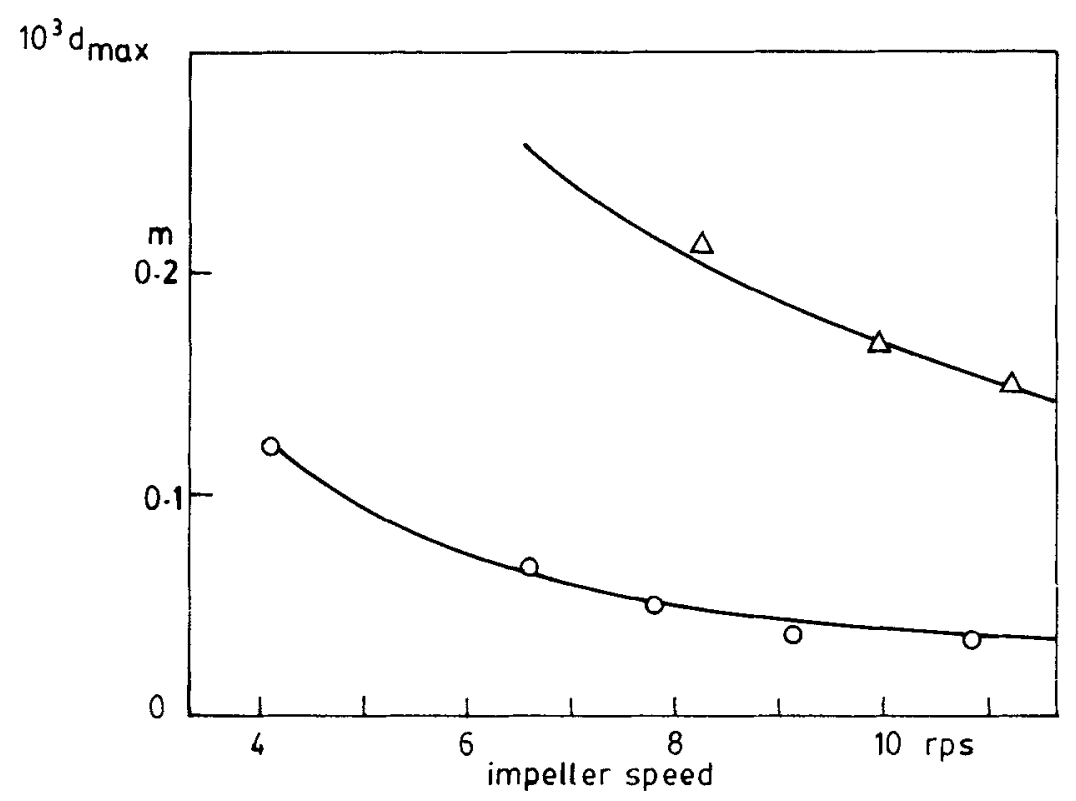

Figure 7. Effect of high concentrations of surfactant on $d_{\operatorname{mux}} . \triangle$ : pure styrene-water system, $\sigma=0.034 \mathrm{~N} / \mathrm{m} ; \mathrm{O}$ : water-Teepol-styrene system, $\sigma=0.0032 \mathrm{~N} / \mathrm{m}$. The solid lines correspond to the model of Koshy et al 1988a, (19).

As the concentration of the surfactant is raised from zero, the $\Delta \sigma$ first increases and then decreases, eventually becoming zero. Figure 7 shows the results for zero and high concentrations of the surfactant (corresponding to $\Delta \sigma=0$ ). It is interesting that the experimental results are explained by the model of Lagisetty et al (1986) which is a special case of the model represented by (19). When a very viscous dispersed phase is used, the effect of surfactant is found to be insignificant, as the breakage is controlled mainly by the viscous stresses, with minor changes in interfacial tension making negligible contribution.

2.2d Mildly viscoelastic dispersed phases: Koshy et al (1988b) conducted experiments on breakage of viscoelastic fluids by using aqueous solutions of polyacrylamide (Separan AP-30) as the dispersed phase. Two solutions having concentrations of 0.25 and $0.5 \%$ were employed. The drop sizes were much larger than those predicted by the model of Lagisetty et al (1986) for purely viscous drops. This model was therefore modified by Koshy et al (1988b). The modification was mainly concerned with the manner in which a viscoelastic drop deforms. It was assumed that the interaction between an eddy and the drop begins sharply. Under these conditions, the polymer acts as a glass and supports the applied stress without significant deformation. As time progresses, the stress borne by the polymer decreases due to the relaxation of the polymer molecules. The deformation therefore proceeds in this manner with the stress borne by the polymer decreasing continuously. The relaxation of the stress borne by the polymer was assumed to follow the expression:

$$
\tau_{p}=\tau_{t} e^{-t / \lambda}
$$

where $\lambda$ is the relaxation time of the polymer. With this modification the equation 


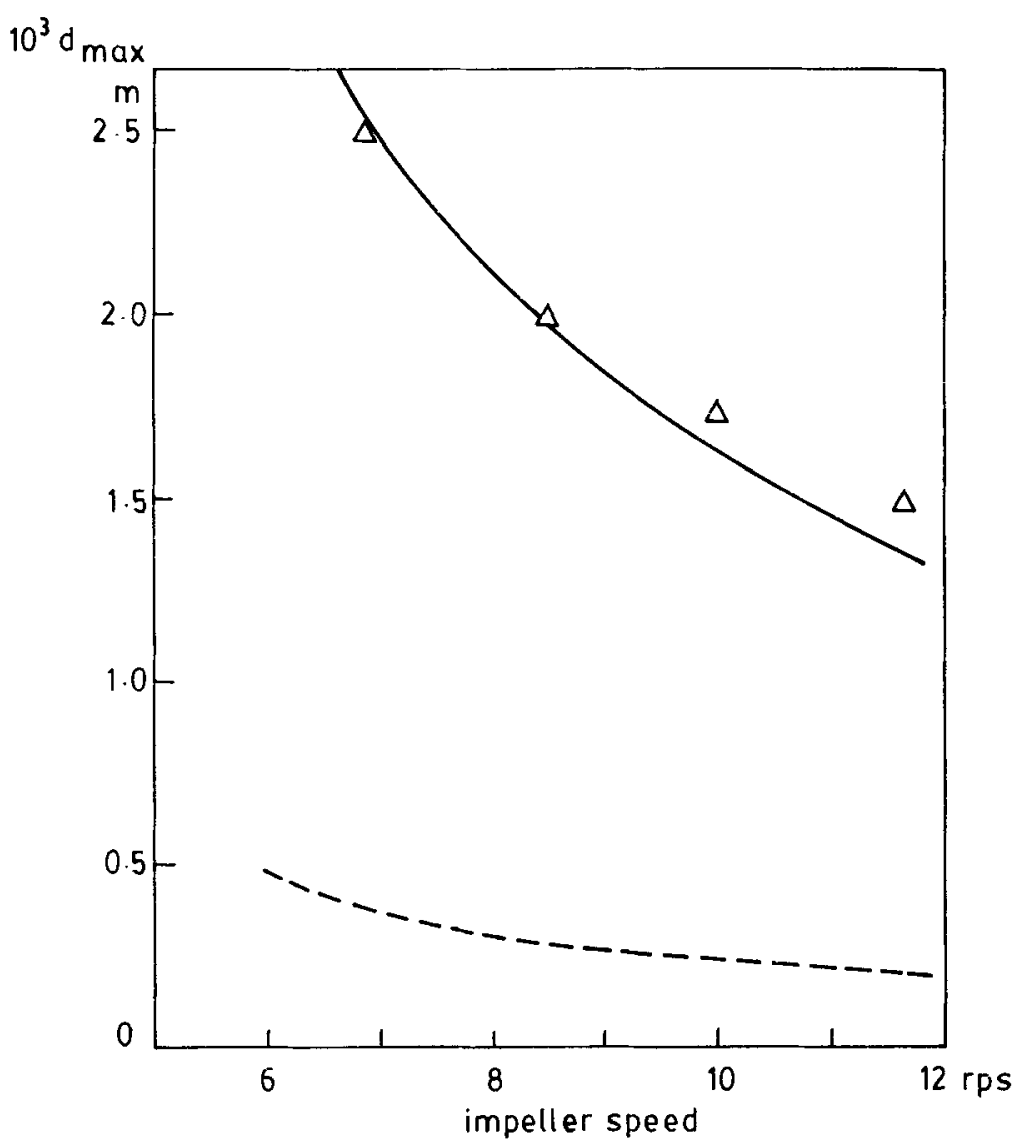

Figure 8. Verification of the model of Koshy et al (1988b) for viscoelastic liquids. System: heptane and carbon tetrachloride (specific gravity 1.06)-0.5\% aqueous solution of polyacrylamide, interfacial tension $=0.057 \mathrm{~N} / \mathrm{m} . \Delta$ experimental points. $-(21) ;-.-$ (18).

for viscoelastic dispersed phases becomes:

$$
\begin{gathered}
C \rho_{\mathrm{c}} N^{2} D^{4 / 3} d^{2 / 3}+\tau_{0}=(\sigma / d) \theta(1-\theta)+\left(C \rho_{\mathrm{c}} N^{2} D^{4 / 3} d^{2 / 3}\right) e^{-t / \lambda}+ \\
+K(\mathrm{~d} \theta / \mathrm{d} t)^{n}
\end{gathered}
$$

The $d_{\max }$ can be found as before by integrating the above equation with the initial and breakage conditions described earlier. The verification of the model has been shown in figure 8 , where $d_{\max }$ is plotted as a function of rps for $0.5 \%$ polyacrylamide solution as the dispersed phase. The points are experimental whereas the solid line is based on the model. The predictions made by the model of Lagisetty et al (1986) are shown as the dotted line, and fall significantly below the observed values of $d_{\max }$. The above model has been found to successfully predict the $d_{\max }$ values only when the dispersed phase is mildly viscoelastic (having time constants of the order of $0.1 \mathrm{~s}$ or less). For highly viscoelastic dispersed phases, no drop breakage was observed experimentally. Instead, the dispersed phase was found to be present in the form of big blobs which elongated into thread-like structures when they passed through the impeller zone. The model was unable to predict such behaviour. 
2.2e Breakage in presence of drag-reducing agents: A number of investigators (Mashelkar et al 1975; Quraishi et al 1976; Hoecker et al 1980) have shown that the addition of small quantities of drag-reducing agents (DRA) to a stirred vessel results in torque suppression, indicating that the turbulent energy pattern gets altered. Thus, the drops formed in such a situation, should be larger than those obtained without the addition of DRA. Walstra (1974) measured average drop size of paraffin oil in a turbomixer by adding polyvinyl alcohol (PVA) to the continuous water phase and found that with increase in PVA concentration, the drop size first decreased and then increased. However when a surfactant was also added, the average drop size increased with increase in PVA concentration. Koshy et al (1989) measured $d_{\max }$ values for a number of systems and found that the addition of even $25 \mathrm{ppm}$ of polyacrylamide to the continuous aqueous phase resulted in an increase in $d_{\max }$ values. They did not use any surfactant in the continuous phase in their experiments. They were able to predict the increase quantitatively by considering that the DRA change the energy budget of the eddies thereby causing a change in the inertial stress. In the elongating eddies, the DRA molecules elongate and store a part of the eddy energy as potential energy. On drop-eddy interaction, the molecules relax releasing a part of the stored energy. The energy available for breakage can be obtained by subtracting the stored energy at any time from the energy of the eddy without drag-reducing agents. Thus

$$
\tau_{t}=C\left[\rho_{c} \overline{u^{2}(d)}-\left(e_{p s}-e_{p r}\right)\right]
$$

The energy stored by a polymer molecule was evaluated by the finitely extendable elastic dumbbell (FENE) model. The molecule extends because of the difference in velocity across the molecule, but this extension is retarded because of the elastic spring. Finally, the extended molecule reaches an equilibrium size corresponding to the strain rate existing in the eddy. The energy of an extended molecule is:

$$
E_{p}\left(R_{0}, d\right)=f\left(H R_{0}^{2} / 2\right) \ln [1+(\zeta / H \bar{T})] .
$$

This equation has to be modified by multiplying the right hand side with $d / l_{\mathrm{T}}$ if the eddy size is smaller than the Taylor microscale, $l_{\mathrm{T}}$.

As there will be a molecular weight distribution of the polymer molecules, the overall energy accumulated in all the polymer molecules present per unit volume works out to be:

$$
e_{p s}=(3 / 2)(f / n)\left(\rho_{c} R_{g} T / m_{0}\right) w \int_{0}^{\infty} g(p) \ln \left[1+\left(4 \pi^{2} \lambda_{0} / 6 \bar{T}\right) \mathrm{d} p\right.
$$

The above expression also has to be multiplied by $d / l_{T}$ if the eddy size is smaller than the Taylor microscale, $l_{\mathrm{T}}$. For evaluating the energy released back by the polymer molecules to the eddy, they used the expression:

$$
E_{p}\left(1-e^{-\bar{T} t_{i 1}}\right)
$$

Taking the molecular weight distribution into account, $e_{p r}$ was found to be:

$$
e_{p r}=(3 / 2)(f / n)\left(\rho_{c} R_{g} T / m_{0}\right) w \int_{0}^{x} g(p)\left(1-e^{-\bar{T} / j_{0}}\right) \ln \left[1+\left(4 \pi^{2} \hat{\lambda}_{0} / 6 \bar{T}\right)\right] \mathrm{d} p
$$




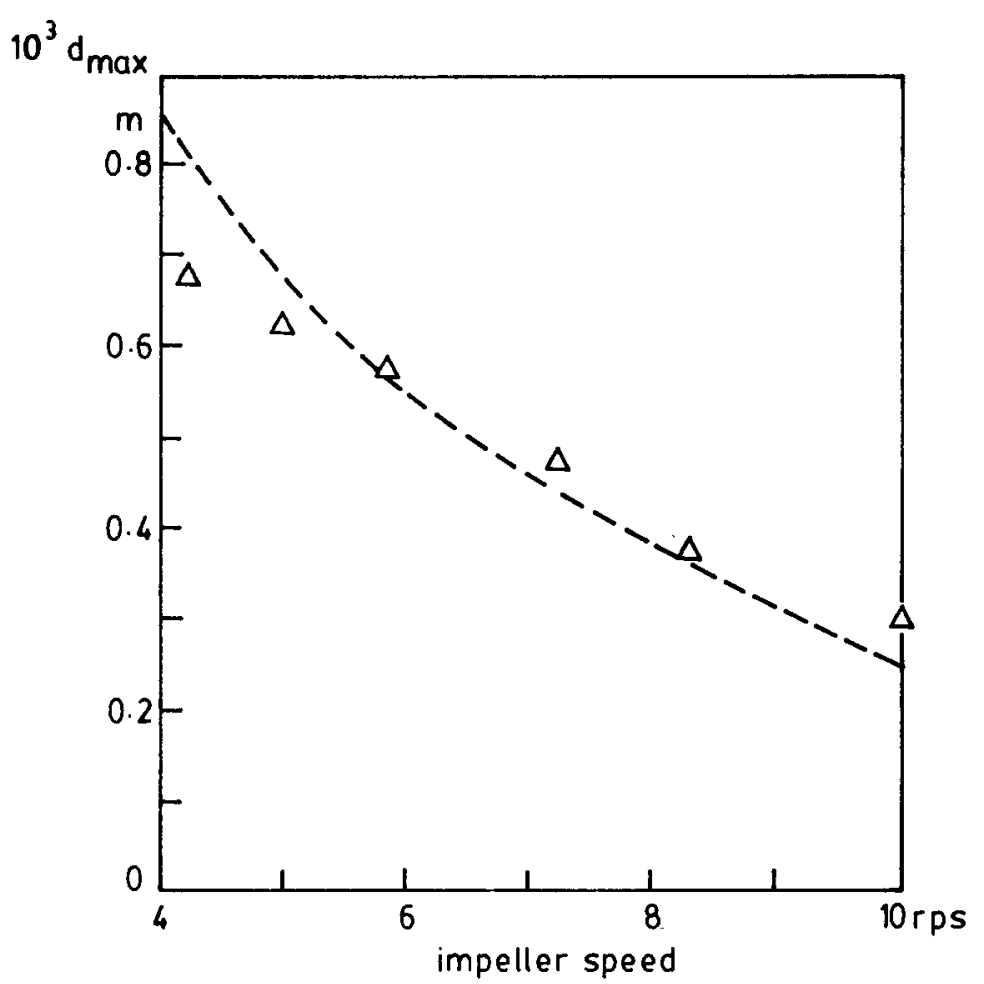

Figure 9. Effect of a drag reducing agent on $d_{\max }$. System: toluene-50 ppm aqueous solution of polyacrylamide, interfacial tension $0.036 \mathrm{~N} / \mathrm{m}$. $\Delta$ experimental points; —- model of Koshy et al (1989), (28).

Substitution of expressions for $e_{p r}$ and $e_{p s}$ in (22) yields:

$$
\tau_{t}=8 \rho_{c} \overline{u^{2}(d)}-12(f / n)\left(\rho_{c} R_{g} T / m_{0}\right) w \int_{0}^{\infty} g(p)\left(e^{-\bar{T} / \dot{j}_{0}}\right) \ln \left[1+\left(4 \pi^{2} \lambda_{0} / 6 \bar{T}\right)\right] \mathrm{d} p .
$$

The rest of the analysis of Koshy et al (1989) follows the same lines as that of Lagisetty et al (1986). The final expression for $d_{\max }$ is:

where

$$
(\operatorname{Re} / \mathrm{We})\left(d_{\max } / D\right)^{-1 / 3}=\left(4 / \alpha^{\frac{1}{2}}\right) \tan ^{-1}\left(1 / \alpha^{\frac{1}{2}}\right),
$$

$$
\alpha=32\left[\mathrm{We}-\left(\tau_{u} d_{\max } / \sigma\right)\right]\left(d_{\max } / D\right)^{5 / 3}-1,
$$

and $\tau_{u}$ is the last term of (27). Figure 9 presents a typical comparison between the predicted and experimental values of $d_{\max }$ as a function of rps for a water-toluene (dispersed) system, when $50 \mathrm{ppm}$ of polyacrylamide were added to the continuous (water) phase. It is possible to explain the results obtained for other systems also including the ones containing surfactants, when DRA are added to the continuous phase.

2.2f Variation of $d_{\max }$ with dispersed phase volume fraction: The turbulence in the stirred vessel is dampened as the volume fraction of the dispersed phase (hold-up) increases. Though there is no theory available to predict the dampening quantitatively, 
empirical expressions have been proposed to calculate the effect. Typical of such correlations is the following proposed by Laats \& Frishman (1974):

$$
\left[\overline{u^{2}(d)}\right]_{\phi=\phi}=(1+4 \phi)^{-2}\left[\overline{u^{2}(d)}\right]_{\phi=0}
$$

Lagisetty et al (1986) have shown that by modifying the turbulent stress in accordance with the above empirical expression, the effect of the hold-up of the dispersed phase on the values of $d_{\max }$ can be predicted up to a hold-up value of about $0 \cdot 3$.

$2.2 \mathrm{~g}$ Some outstanding problems: It is seen that our understanding of drop breakage in turbulent stirred dispersions is still at a rudimentary stage. First generation models, which bypass the flow field and simplify the three-dimensional problem to a unidimensional one are however available. These models, though gross, can predict the $d_{\max }$ values for lean dispersions containing viscous drops, though they fail when the dispersed phase is highly viscoelastic or its viscosity is extremely high.

In slightly concentrated dispersions, the turbulence gets suppressed and empirical modifications have to be resorted to for the prediction of $d_{\max }$. Even these work only up to a dispersed phase hold-up $(\phi)$ of about 0.3. Beyond this dispersed phase hold-up, highly unexpected results are obtained. With increased hold-up, the turbulence intensity decreases. Thus the $d_{\max }$ value is expected to rise as $\phi$ is increased. This however happens only up to a $\phi$ of around $0 \cdot 3$, as is seen from figure 10 , which presents $d_{\max }$ as a function of $\phi$ for the water-toluene system. A surfactant was added to the continuous phase to suppress coalescence, thus making the process predominantly breakage controlled. It is seen from the figure that beyond a value of $\phi$ of around 0.3 , the $d_{\max }$ values decrease instead of increasing. These results lead one to speculate that under such conditions, the $d_{\max }$ is not decided by the turbulent breakage mechanism as this would yield an increase in $d_{\max }$. Instead $d_{\max }$ is now decided by other mechanisms. One possibility is that the drops break in the extensional flow generated at the impeller by the liquid approaching the impeller in the middle and accelerating outwards. The second possibility is the drop breakage in the boundary layer existing at the impeller. Here shear breakage would be predominant. Though the magnitudes of elongational rates and shear rates are different, both mechanisms yield a lowering of drop size with $\phi$ which is in qualitative agreement with observed trends. Thus it is likely that the experimentally observed $d_{\max }$ is the minimum of the $d_{\max }$ values generated by these different (viz. turbulent, elongational flow and shear flow) mechanisms. Further conceptual complications arise in the case of concentrated suspensions. For instance, in such media, should suspension viscosity be used for characterizing the continuous phase? Further, what modifications are needed in the theory if the viscosity of the dispersion is such that the tip Reynolds number is reduced below $10^{4}$ ? A reduction by a factor of ten, which would imply that flow is still turbulent, is perhaps the case in most emulsifiers.

The models discussed above have accounted for the breakage occurring in the impeller zone since that is relevant for calculation of $d_{\max }$. However, the breakage that occurs in other areas of the stirred vessel, where shear forces may also be simultaneously important, would also be needed for calculating the drop-size distributions.

Apart from these lacunae, there remain several other conceptual questions. The above models do not really account for the eddy-drop interaction in a mechanistic way. Further, they adopt an arbitrary breakage condition. Thus, does the drop break 


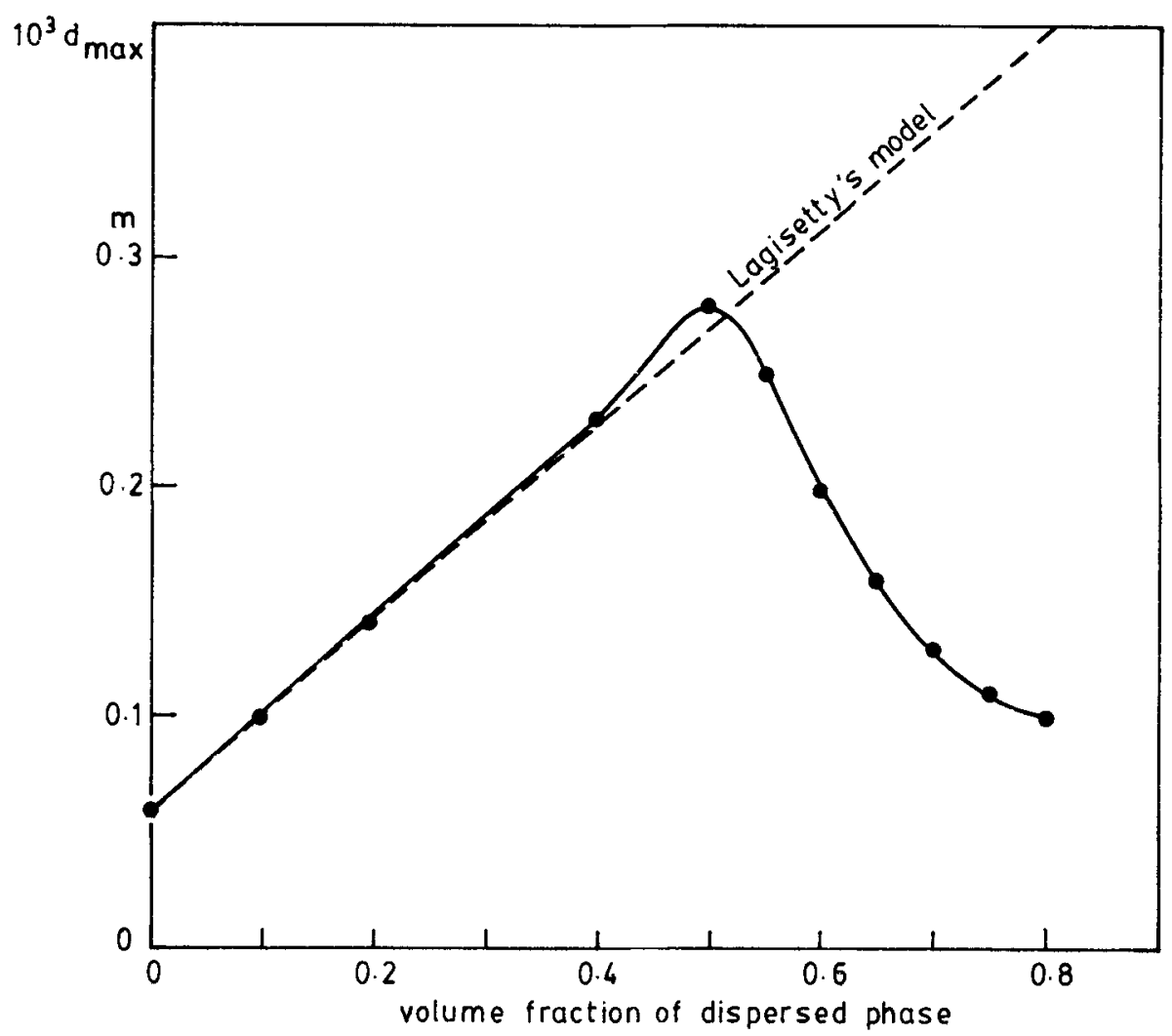

Figure 10. Variation of $d_{\max }$ with dispersed phase hold-up. The dispersed phase is toluene. The continuous phase (water) contains $0.3 \%$ sodium lauryl sulphate to suppress coalescence. The solid line through the experimental points diverges from the model predictions of Lagisetty et al (1986) beyond a hold-up of 0-3.

by surface tension driven instability or by the end-pinching mechanism observed in laminar flows or by an entirely different process? The black box approach towards drop-eddy interaction is also silent on the details of the nature of the flow that occurs inside the drop. For instance, is it elongational or shear in nature? An answer to this question may hold the key to explaining the failure to disperse highly viscoelastic dispersed phases.

Finally it would be interesting to see if the model of Lagisetty et al (1986) can be used in other equipment, such as pulsed columns, nozzles etc. by making appropriate substitution for $\varepsilon$, the power dissipation per unit mass.

Thus even for $d_{\max }$ evaluation, there is need for more robust models which could account for drop-eddy interaction in a more acceptable fashion, take detailed flow field into account and incorporate a more realistic breakage condition.

\subsection{Breakage frequency}

The evaluation of $d_{\max }$ though useful for calculating the average drop diameter existing in the vessel, cannot yield the drop-size distribution. To obtain this, it is necessary to solve the population balance equations, which in turn require expressions for breakage frequency, coalescence frequency and the size distribution of the daughter droplets formed through the breakage of a larger drop. 
Breakage frequency $[\Gamma(v)]$ can be physically interpreted as the fraction of drops of a given size $v$ that break in unit time when they are kept in a turbulent flow field. In view of the time invariance of the statistical properties of the turbulence, it is also a measure of the transitional breakage probability of one such drop i.e. $\Gamma(v) \mathrm{d} t$ is the probability of a drop of size $v$ undergoing breakage in a small time interval $\mathrm{d} t$. Its average value for a given drop size may, therefore, be computed as the reciprocal of the expected survival time of such a drop in the turbulent environment under consideration. A reliable model for this is necessary to enable us to solve the population balance equation and obtain the drop-size distribution. Models are available in the literature which try to predict breakage frequency for inviscid dispersed phases, which undergo instantaneous breakage. There is no reported model which attempts to predict breakage frequency for dispersed phases which have finite viscosities or which display more complex rheological behaviour.

2.3a Models for breakage frequency: Early models of breakage frequency have been either ad hoc or have drawn upon analogies to rate expressions characteristic of chemical reactions. Thus Valentas \& Amundson (1968) have assumed the breakage frequency (regarded as a function of the drop diameter, $d$, in this section) to be proportional to the droplet surface area:

$$
\Gamma(d)=C_{1} d^{2}
$$

Ross \& Curl (1973) saw a similarity between drop breakage and chemical decomposition. They assumed a drop to form an 'activated complex' due to imparted kinetic energy, which then broke just like a chemical reaction. Thus:

$$
\text { normal drops } \rightleftharpoons \text { 'activated drops } \stackrel{ }{ }{ }^{\prime \prime} \rightarrow \text { breakage products, }
$$

where the normal drops are in equilibrium with the activated drops and $K^{\prime \prime}$ is the rate constant for the decomposition or the break-up process. The breakage frequency is thus simply

$$
\Gamma(d)=K^{*} K^{\prime \prime}
$$

where $K^{*}$ is the equilibrium constant for the normal-activated drop exchange. From similarity to molecular decomposition,

$$
K^{*}=\exp (- \text { activation energy/kinetic energy). }
$$

The kinetic energy is assumed to come from all eddies smaller than the drop since larger eddies would be expected to convect the drop without breaking it. The activation energy is taken to be proportional to $\sigma d^{2}$ where $\sigma$ is the interfacial tension. Their final expression is:

$$
\Gamma(d)=C_{1} N D^{2 / 3} d^{-2 / 3} \exp \left[-C_{2} \sigma /\left(\rho_{c} N^{2} D^{4 / 3} d^{5 / 3}\right)\right]
$$

The two unknown parameters $C_{1}$ and $C_{2}$ are determined from the experimental data to obtain the best fit.

Coulaloglou \& Tavlarides (1977) define the breakage frequency on more physical grounds as:

$$
\Gamma(d)=(1 \text { breakage time }) \times \begin{gathered}
\text { number fraction of drops of size } d \\
\text { breaking in that time. }
\end{gathered}
$$


They assumed that the fraction of drops breaking is proportional to the fraction of drops of size $d$ which have a total kinetic energy greater than a minimum value necessary to overcome the surface energy holding the drop intact. This minimum surface energy was taken as

$$
E_{\mathrm{c}} \propto \sigma d^{2}
$$

Using the two-dimensional normal distribution for the velocity fluctuations of the eddies, they derived an expression for the fraction of eddies with kinetic energies exceeding $E_{c}$ :

$$
\text { fra mon of eddies with } E>E_{c}=\exp \left[-\left(E_{c} / E_{t}\right)\right] \text {. }
$$

Here $E_{t}$ is the kinetic turbulent energy of an eddy of size $d$ and is related to the energy dissipation per unit mass by

$$
E_{t} \propto \rho_{c} \varepsilon^{2 / 3} d^{11 / 3}
$$

They estimate the time available for breakage to be

$$
t_{b}=d^{2 / 3} \varepsilon^{-1 / 3}
$$

from consideration of the relative motion of two lumps of fluid in a turbulent flow field as described by Batchelor (1953). In terms of the operating parameters of the stirred vessel, their final expression is:

$$
\Gamma(d)=K_{1} d^{-2 / 3} N D^{2 / 3} \exp \left[-K_{2} \sigma /\left(\rho_{c} D^{4 / 3} N^{2} d^{5 / 3}\right)\right],
$$

where $K_{1}$ and $K_{2}$ are parameters to be determined from experimental data. As is evident, the dispersed phase viscosity has been ignored in the development of the breakage frequency expression. In addition, the idea of time required for the centres of mass of the would-be daughter droplets to separate may have little to do with the breakage phenomenon because during fragmentation itself the parent drop is quite deformed and would give rise to already separated daughter droplets.

Narsimhan et al (1979) view the droplet as a one-dimensional simple harmonic oscillator, oscillating about its spherical equilibrium shape. Oscillations are induced by the arrival of eddies of different energies at the surface of the droplet. They argue that the increase in the surface energy required for fragmentation is minimum if binary equal breakage occurs and has the value

$$
\left(2^{1 / 3}-1\right) \sigma \pi^{1 / 3} 6^{2 / 3} v^{2 / 3}
$$

for a drop of volume $v$. The arrival of eddies at the surface of a drop is assumed to be a Poisson process with a parameter $\lambda$ independent of the droplet diameter. The arriving eddy has a kinetic energy per unit mass of $\frac{1}{2} u^{2}$ where $u$ is an associated characteristic velocity. To be able to break the drop, therefore, one must have

$$
\frac{1}{2} u^{2} \geqslant\left(2^{1 / 3}-1\right) \sigma \pi^{1 / 3} 6^{2 / 3} v^{2 / 3} /\left(\rho_{c} v\right) \text {. }
$$

Assuming that the joint probability distribution of 2 point-velocities in an agitated vessel is normal with a variance

$$
\sigma^{2}=2(\varepsilon d)^{2 / 3},
$$


they arrive at the following expression for the breakage frequency

where

$$
\Gamma(d)=\lambda \operatorname{erfc}\left[\sqrt{a}(\pi / 6)^{-1 / 6} d^{-5 / 6} /\left(2 \varepsilon^{-1 / 3}\right)\right]
$$

$$
a=2\left(2^{1 / 3}-1\right) \pi^{1 / 3} 6^{2 / 3}\left(\sigma / \rho_{c}\right) .
$$

Das (1984) points out that even though the drop gets to interact with an eddy of sufficiently high energy, the breakage itself need not be instantaneous. Furthermore, there is a finite probability of the nascent daughter droplets recombining which has not been accounted for in the above model. He has modified this model by considering the possibility of recombination of the daughter droplets also.

None of the foregoing models addresses the situation wherein the dispersed phase has a finite, non-zero viscosity. As the breakage of drops of size $d_{\max }$ is not possible, the size distributions generated by these models contain drops predominantly in the region of $d_{\max }$. Experimentally obtained distributions however contain drops much smaller than $d_{\text {max }}$. Furthermore, they yield no information about the daughter droplet distribution $\beta\left(v, v^{\prime}\right)$ and subsequent use of these breakage models in the population balance equation has to be coupled with independent assumptions about $\beta\left(v, v^{\prime}\right)$. In the absence of a phenomenological model, it has been the practice to assume expressions for $\beta\left(v, v^{\prime}\right)$ that render the population balance equations analytically tractable. Thus, uniform breakage $\left[\beta\left(v, v^{\prime}\right)=1 / v^{\prime}\right]$, equal breakage $\left[\beta\left(v, v^{\prime}\right)=\delta\left(v-v^{\prime} / 2\right)\right]$ and 'normal' breakage $\left\{\beta\left(v, v^{\prime}\right)=\left[\sigma(2 \pi)^{1 / 2}\right]^{-1} \exp \left[-\left(v-v^{\prime} / 2\right)^{2} / 2 \sigma^{2}\right]\right\}$ have been reported in the literature (Narsimhan et.al 1979; Shiloh et al 1973; Coulaloglou \& Tavlarides 1977).

Nambiar and others (D K R Nambiar, T R Das, R Kumar \& K S Gandhi, unpublished) have recently developed a model which not only predicts the breakage frequency $[\Gamma(v)]$, but also $\beta\left(v, v^{\prime}\right)$. These authors consider the possibility of unequal breakage of drops larger than $d_{\max }$. They find that once the drop's diameter has a value of $d_{\max }$ or lower, it cannot be further broken. However larger drops can undergo unequal breakage yielding very small drops which are observed in actual experiments. It is found that for a drop of given size, there is a range of eddy sizes whose interaction with the drop can result in its breakage. The size range of eddies $\left(l_{\min } \leqslant l \leqslant d\right)$ capable of breaking a drop of size $d$ has been obtained through a slightly modified version of the model of Lagisetty et al (1986). The variation of $l_{\min }(d)$ with the drop diameter, $d$, is shown in figure 11 . It is seen that for $d_{\max }$, the ratio of $l_{\min }(d) / d$ is unity, indicating that smaller eddies cannot break the drop. To obtain the breakage frequency, the drop is permitted to interact sequentially with eddies of various sizes existing in the vessel. If the drop interacts with an eddy much larger in size than itself, it will be simply convected and no breakage will result. Similarly if an eddy of size lesser than $l_{\text {min }}(d)$ interacts with it, the drop will get slightly deformed but will not break. It is only when an eddy having size greater than $l_{\text {min }}(d)$ and less than $d$ interacts with it that breakage will occur. The eddy-size distribution evaluated on the basis of the energy cascade hypothesis is

$$
f(l)=2\left(D l_{K}\right)^{2} /\left[\left(D^{2}-l_{K}^{2}\right) l^{3}\right] .
$$

With this eddy size distribution, the expression for the expected survival time of a drop, $t_{e x}$, works out to be:

$$
t_{e x}=(q / p) \int_{l_{k}}^{D} \bar{T}(l) f\left[l \mid l \notin\left(l_{\min }, d\right)\right] \mathrm{d} l+\int_{l_{k}}^{D} t_{b}(l, d) f\left[l \mid l \in\left(l_{\min }, d\right)\right] \mathrm{d} l,
$$




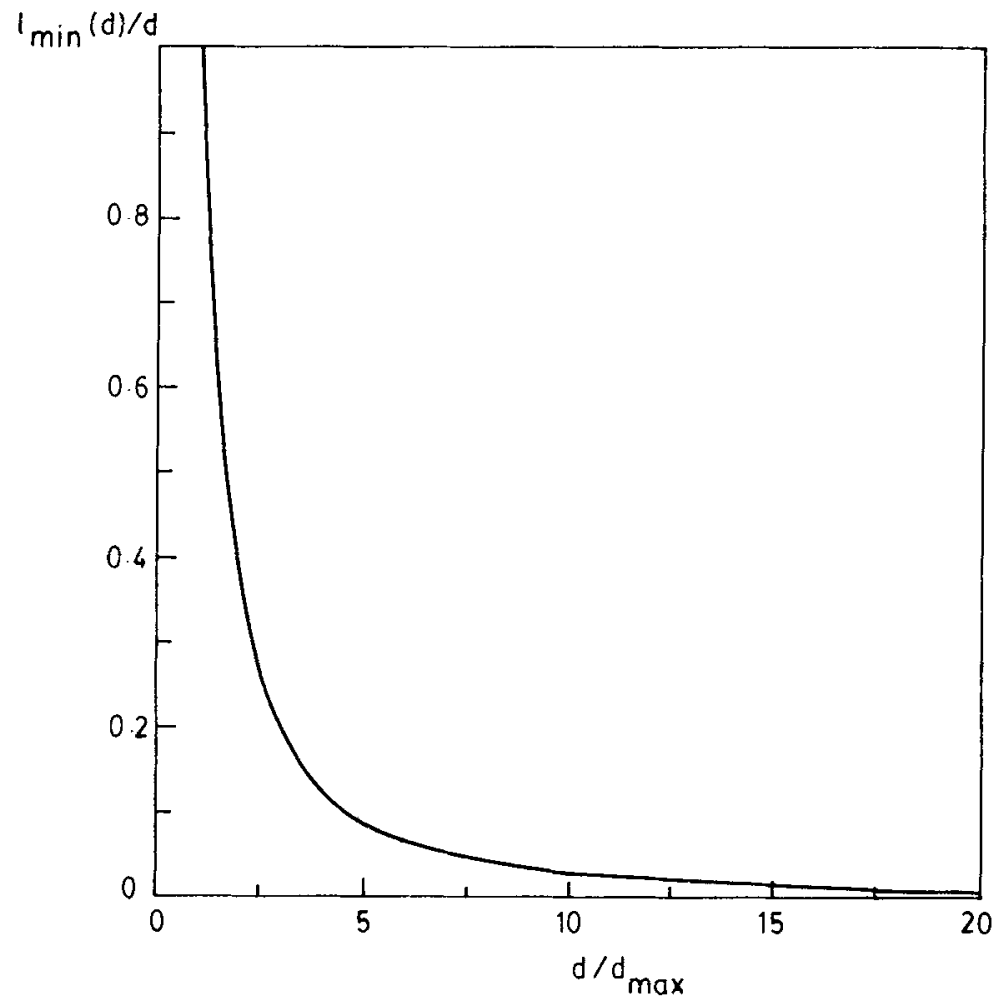

Figure 11. Variation of $l_{\min }(d)$ with drop diameter $(d)$.

where

and

$$
p=\int_{l_{\min }}^{d} f(l) \mathrm{d} l,
$$

$$
q=1-p
$$

The breakage frequency is obtained as the reciprocal of the expected survival time,

$$
\Gamma(v)=1 / t_{\text {ex }}
$$

A typical comparison of the variation of the breakage frequency with drop size with the variation predicted through (49) is shown in figure 12. The effect of raising the dispersed phase viscosity hundred-fold has also been indicated in the figure. The breakage frequency tends to be lower in this case. Figure 13 shows the results of a numerical solution of the population balance equation incorporating the above expression, (49), for $\Gamma(v)$ and neglecting coalescence. The initial population in this case consists of uniformly sized particles of size $10 d_{\max }$. The cumulative volume fraction evolves until no drops of size exceeding $d_{\max }$ survive in the system. The solid line in figure 13 shows the steady state size distribution obtained experimentally by Narsimhan et al (1980) using an encapsulation technique. It is difficult to describe the initial size distribution in experiments on a batch system to any degree of accuracy and this makes comparison of transient size distributions with numerical solutions difficult. It is reasonable to believe, though, that the steady state size distribution should be independent of the initial conditions. 


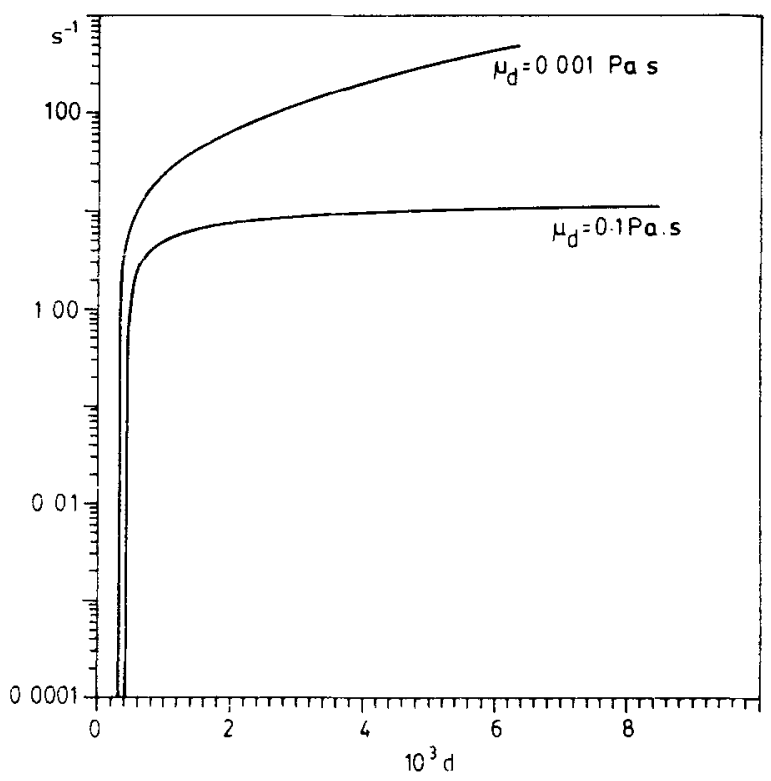

Figure 12. Variation of the breakage frequency $(\Gamma)$ with drop diameter $(d)$. The effect of increasing the dispersed phase viscosity $\left(\mu_{d}\right)$ is also indicated.

The daughter droplet distribution may be obtained directly from the eddy size distribution using the geometric relation between the daughter droplet volume $v^{\prime}$ and the size $l$ of the eddy-causing breakage (Papoulis 1984, p. 95).

$$
\beta\left(v^{\prime}, v\right)=\frac{1}{2}\left\{f\left(l \mid l_{\min } \leqslant l \leqslant \mathrm{~d}\right)\right\} /\left\{\left|\left(\mathrm{d} v^{\prime} / \mathrm{d} l\right)\right|\right\} .
$$

If the angle $\psi$ is defined by

$$
\cos (\psi)=1-\left(2 v^{\prime} / v\right)
$$

the final expression for $\beta\left(v^{\prime}, v\right)$ becomes

$$
\beta\left(v^{\prime}, v\right)=\left\{8\left(D l_{K}\right)^{2} /\left[p \pi\left(D^{2}-l_{K}^{2}\right) l^{4} d\right]\right\}[\sin |(\pi / 3)-(2 \psi / 3)| / \sin \psi]
$$

It is seen from this expression that for binary breakage, equal breakage, which has been hitherto a standard assumption, is more of an exception than the rule.

2.3b Comments on breakage frequency models: Breakage frequency is not measured directly but is extracted from the evolution of the drop size distribution with time in a stirred vessel, under conditions where breakage is predominant. This is normally achieved through using low dispersed phase hold-up. The method of extraction of breakage frequency information from the evolving drop size distribution has been described by Narsimhan et al (1984). The existing models of breakage frequency have not been rigorously tested even when the dispersed phase viscosity is low. For dispersed phases of high viscosity, the only model is that of Nambiar and others (D K R Nambiar, T R Das, R Kumar \& K S Gandhi, unpublished) and it has not been tested against any experimental data, as such data are not available. Similar is the 


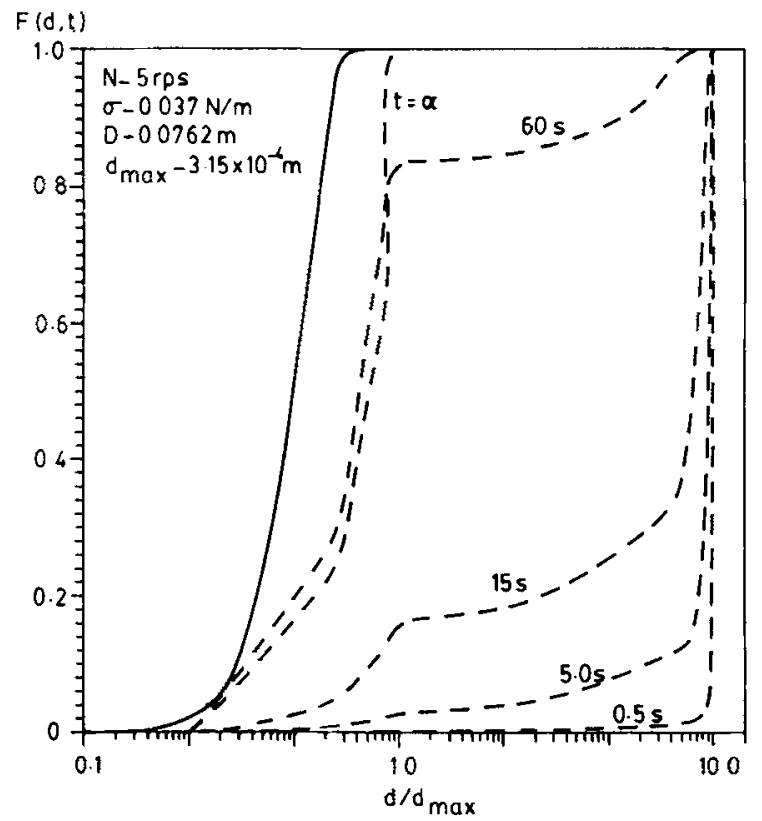

Figure 13. Numerical solution of the population balance equation showing the evolution of the cumulative volume fraction $F(d, t)$ with time. $F(d, t)$ is the contribution at time $t$ to the dispersed phase volume fraction from drops of diameter not exceeding $d$. The initial dispersion is monodisperse particles of size $10 d_{\max }$. The solid line shows the steady state size distribution obtained experimentally by Narsimhan et al (1980) for the same parameter values.

situation for dispersed phases displaying more complex rheological behaviour. Thus there is need for experimental information so that existing models can be tested and new models developed if necessary.

When the dispersed phase hold-up becomes high, other mechanisms of breakage involving flow at the impeller become important. For such breakage mechanisms, there are no models available for breakage frequency and entirely new models are required to be developed.

Expressions for $\beta\left(v, v^{\prime}\right)$ are normally assumed. Equation (52) is the only expression based on a mechanistic model. At present, it is not possible to recommend any particular expression for $\beta\left(v, v^{\prime}\right)$ as the experimental measurement of $\beta\left(v, v^{\prime}\right)$ has not been possible.

\subsection{Coalescence frequency}

The other most important term required for the solution of the population balance equation is the coalescence frequency. Its prediction involves an analysis of the relative motion between drops. For two drops to coalesce, they must first come very close to each other and then the intervening liquid film between them must drain to such a thickness that it becomes unstable and ruptures. Therefore the problem is normally approached through a two-step procedure. Collision frequency is first computed from an estimation of the diffusion coefficients of different droplets in an isotropic turbulent field. This is then multiplied by coalescence efficiency to account for the complex hydrodyn.mic interactions that are ignored in the first step. 
2.4a Models for collision frequency: Smoluchowski (1917), and later Harper (1936) have considered the problem of determining the collision frequency of spherical particles subject to thermal agitation. Two particles of radii $r_{1}$ and $r_{2}$ may be said to be in collision when the distance between their centres is $s=r_{1}+r_{2}$. According to the theory, if $D_{1}$ and $D_{2}$ be the Brownian diffusivities of the two kinds of particles in a binary mixture wherein their number densities are $n_{1}$ and $n_{2}$ respectively, the frequency of collisions between them per unit volume of the system is given by

$$
v^{\prime}=4 \pi n_{1} n_{2} s\left(D_{1}+D_{2}\right) \text {. }
$$

Howarth (1964) reasoned that the above expression could also be used to predict the frequency of collisions between spherical particles suspended in an isotropic, turbulent field, it only being necessary to account for the enhanced diffusivity that is characteristic, in general, of turbulence. For isotropic turbulence, when the diffusion times are short in relation to the Lagrangian integral time scale, the diffusion coefficient is time dependent and is given by (Taylor 1921)

$$
D_{t}=\overline{u^{2} t}
$$

where $\overline{u^{2}}$ is the mean square Lagragian turbulent velocity fluctuation. The time available for diffusion is of the order of the reciprocal of the collision frequency. Thus Howarth (1964) estimates the frequency of collision as

$$
v_{C}=\left(4 \pi n s \bar{u}^{2}\right)^{\frac{1}{2}} .
$$

An alternative approach to the problem of inter-particle collisions in a turbulent medium is supplied by the kinetic theory of gases. The droplets under consideration here are larger than the microscale of turbulence. They are not therefore completely entrained by the turbulent eddies. The impact of eddies on them in all directions causes them to movein a random fashion mimicking the random motion of molecules in an ideal gas. Based on this concept, Coulaloglou \& Tavlarides (1977) provide the following expression for the collision frequency between drops of volume $v$ and $v^{\prime}$ :

$$
v\left(v, v^{\prime}\right)=\left(9 \pi^{\frac{1}{3}} / 2\right)\left(v^{\frac{2}{3}}+v^{\prime \frac{2}{3}}\right)\left[\overline{u^{2}(v)}+\overline{u^{2}\left(v^{\prime}\right)}\right]^{\frac{1}{2}} .
$$

The above expression is typical of this approach and variations, differing from the above only marginally, have been proposed by several other workers (Rietema 1964; Kuboi et al 1974; Abrahamson 1975).

Both the above approaches to collision frequency are extensions of the existing theories to domains outside the regime of their strict validity. The diffusing elements in Taylor's theory are indistinguishable from the fluid and extension of this concept to macroparticles that alter the flow field around them is open to argument. The kinetic theory models, on the other hand, are intuitive generalizations and their validity comes into serious question when extended to turbulence in a stirred vessel. However, in the absence of more realistic models, one of the models described above is normally employed.

2.4b Models for coalescence efficiency: The models for the collision frequency clearly view the droplets as hard spheres consequently ignoring th finer details of droplet deformation, the thinning of the intervening film and its rupture at a critical value of thickness. These details are considered in defining the coalescence efficiency which estimates the proportion of collisions that actually result in coalescence. 
Howarth (1964) has developed an expression for the coalescence efficiency by deeming coalescence between two drops to occur if the relative velocity along the line of their centres exceeds a critical value. By assuming that the three-dimensional Maxwell's equation describes the drop turbulent velocity fluctuations, he obtained the coalescence efficiency as the fraction of drops which have kinetic energy exceeding the critical value $W^{*}$. Thus the coalescence efficiency between drops of volume $v$ and $v^{\prime}$ is given by

$$
\eta=\exp \left(-3 W^{*} / 4 \overline{u^{2}}\right)
$$

where $\overline{u^{2}}$ is the mean square turbulent velocity fluctuation.

Coulaloglou \& Tavlarides (1977) have assumed the following form for the coalescence efficiency:

$$
\eta=\exp \left(-\bar{t}_{c} / \bar{t}\right)
$$

Here $\bar{t}_{c}$ and $\bar{t}$ are the mean coalescence time and the mean contact time respectively. The mean contact time is estimated by viewing the coalescing droplet pair as being entrained in an eddy of length scale $d$, where $d$ is the sum of the diameters of the coalescing droplets. The contact time is then the mean lifetime of such an eddy,

$$
\bar{t}=\varepsilon^{1 / 3} d^{2 / 3} .
$$

The coalescence time $\bar{t}_{c}$ is the time taken for the intervening liquid film to drain from an initial thickness $h_{0}$ to critical film thickness $h_{c}$ under the action of a net squeezing force $F$ effected by the turbulent environment. For drainage between deformed spheres, Chappelear (1961) gives the drainage time as

$$
t-t_{0}=\left(3 \mu_{c} F / 16 \pi \sigma^{2}\right)\left[\left(1 / h^{2}\right)-\left(1 / h_{0}^{2}\right)\right]\left[d d^{\prime} /\left(d+d^{\prime}\right)\right]^{2} .
$$

The force is estimated as

where

$$
F=\rho_{c} \overline{u^{2}}\left[d d^{\prime} /\left(d+d^{\prime}\right)\right]^{2},
$$

$$
\overline{u^{2}}=\varepsilon^{\frac{2}{3}}\left(d+d^{\prime}\right)^{\frac{2}{3}}
$$

Their final expression for the coalescence efficiency is

$$
\eta=\exp \left\{\left(-k \mu_{c} \rho \varepsilon / \sigma^{2}\right)\left[d d^{\prime} /\left(d+d^{\prime}\right)\right]^{2}\right\}
$$

where $k$ is a parameter.

Das (1984) and Das et al (1987) recognize the stochastic nature of the drainage process. The drainage equation is that for the thinning of a film of viscous liquid trapped between two rigid, unequal spheres under the action of a squeezing force $F$ directed along their line of centres. Unlike earlier deterministic models, however, the drainage is treated as a stochastic process. If the autocorrelation time of the force fluctuations is far smaller than the characteristic time of drainage, the fluctuations may be viewed as white noise (Das et al 1987) superimposed on a constant mean value:

$$
F=\bar{F}-\dot{\delta} \sqrt{T} \zeta_{t} .
$$

Here $\zeta_{t}$ is a Gaussian white noise process and $\bar{F}$ and $\delta$ are, respectively, the mean 
and standard deviation of the force $F$. The parameter $T$ is the autocorrelation time of the force fluctuations. With this assumption, the drainage equation

$$
d h / d t=\left(2 h F / 3 \pi \mu_{c}\right)\left[(1 / d)+\left(1 / d^{\prime}\right)\right]^{2}
$$

becomes a stochastic differential equation and is seen to be completely equivalent to the random, one-dimensional Brownian motion of a hypothetical particle in a bounded interval on the coordinate axis of the variable $x=\ln \left(h / h_{c}\right)$ with a steady drift towards the origin. The Fokker-Planck equation describing the evolution of the transitional probability density is

where

$$
D^{*}\left(\partial^{2} p / \partial x^{2}\right)+(\partial p / \partial x)=(\partial p / \partial \tau)
$$

$$
\begin{aligned}
\tau=\alpha \bar{F} t ; \quad \alpha & =\left(2 / 3 \pi \mu_{c}\right)\left[(1 / d)+\left(1 / d^{\prime}\right)\right]^{2}, \\
D^{*} & =\alpha \delta T /(2 \bar{F} / \delta) .
\end{aligned}
$$

and $p=p\left(x, \tau \mid x_{0}, 0\right)=$ probability that the particle is at location $x$ at time $\tau$ given that its initial location was $x_{0}$.

The initial condition is an assertion of the film thickness at the start of the drainage process:

$$
p\left(x, 0 \mid x_{0}, 0\right)=\delta\left(x-x_{0}\right)
$$

The boundary conditions are:

$$
\begin{aligned}
& p\left(0, \tau \mid x_{0}, 0\right)=0, \\
& -\left.D^{*} \frac{\partial p}{\partial x}\right|_{x=x_{0}}=\left.\left(1+k_{m}\right) p\right|_{x=x_{0}} .
\end{aligned}
$$

The first of these corresponds to an absorbing wall at $x=0$ which indicates that rupture is immediate and the coalescence process gets terminated whenever $h=h_{c}$. The second boundary condition assigns to the particle a finite probability of escape from the interval through its right end-point $x=x_{0}$. Physically this implies that the collisional state of the droplet pair may be terminated and the droplet pair may be separated at their initial separation $h_{0}$. If it is further assumed that the collision time is very large in relation to the time scales over which the fate of the particle pair (coalescence or separation) is decided, the efficiency of coalescence is obtained as

$$
\begin{aligned}
\eta & =\text { probability that the Brownian particle will ever be absorbed at } x=0 \\
& =\left.\int_{0}^{x} D_{-}^{*} \frac{\partial p}{\partial x}\right|_{x=0} \mathrm{~d} \tau .
\end{aligned}
$$

For coalescence between drops of diameters $d$ and $d^{\prime}$, the standard deviation of the force fluctuations in the above model has been taken to be

$$
\delta=\rho_{c} \overline{u^{2}\left(d+d^{\prime}\right)}\left[d d^{\prime} /\left(d+d^{\prime}\right)\right]^{2} .
$$

The mean value of the force is assumed to be proportional to $\delta$ with the proportionality constant expected to decrease with increasing intensity of turbulence. The autocorrelation time $T$ has been assigned a constant value of $10^{-4}$. 
Muralidhar \& Ramkrishna (1986) have pointed out that a case for neglecting the dynamic drop deformation during film drainage can be made only after due consideration of the various time scales characteristic of the process. At least the following five different time scales can be identified:

i) film liquid flow, $T_{l}$

ii) drop deformation, $T_{d}$

iii) force fluctuations, $T_{f}$

iv) film thinning, $T_{t}$ and

v) film expansion, $T_{s}$.

Neglecting the dynamic drop deformation amounts to tacitly assuming that

$$
T_{d} \gg T_{f}
$$

In a similar vein, viewing the force fluctuations as a white noise demands that

$$
T_{f} \ll T_{t}
$$

If the force fluctuations have a finite autocorrelation time it is more appropriate to model them as a correlated, band-limited or coloured noise (Muralidhar et al 1988). The time evolution of the force is itself then described by a stochastic differential equation,

$$
\mathrm{d} F^{\prime}=-\left(F^{\prime} / T\right) \mathrm{d} t+\left(\delta / T^{\frac{1}{2}}\right) \mathrm{d} \mathrm{W}_{\imath} .
$$

$W_{t}$ is the standard Wiener process. For rigid spheres, the film drainage equation has the form

$$
\mathrm{d} h=-\alpha h\left(\bar{F}+F^{\prime}\right) \mathrm{d} t .
$$

Defining dimensionless quantities,

and

$$
\begin{aligned}
& \eta=t / T \\
& x=\ln \left(h / h_{c}\right) / \ln \left(h_{0} / h_{c}\right),
\end{aligned}
$$

$$
y=F^{\prime} / \bar{F},
$$

the system of equations describing the drainage process is:

$$
\begin{aligned}
& \mathrm{d} x=\left[\alpha \bar{F} T / \ln \left(h_{c} / h_{0}\right)\right](1+y) \mathrm{d} \eta, \\
& \mathrm{d} y=-y \mathrm{~d} \eta+(\delta / \bar{F}) \mathrm{dW}_{\eta} .
\end{aligned}
$$

The associated initial conditions specify the initial separation $x_{i}$ and the initial force $y_{i}$ which is a random variable drawn from a normal distribution with zero mean and variance $\delta^{2} / 2 \bar{F}^{2}$. The drainage is readily seen to be equivalent to the two-dimensional motion of an abstract particle on the $x-y$ plane confined to the region bounded by the lines $x=0$, representing coalescence, and $x=1$, at which separation occurs. A value of $y>-1$ implies that the force on the drop pair is squeezing in nature and, consequently, a particle hitting the left boundary $x=0$ with such a value of $y$ would coalesce and leave the system. The entire ray $x=0, y>-1$ is thus an absorbing wall. Similar considerations prevail at the right boundary where the ray $x=1, y<-1$ is an absorbing barrier for particles exiting the coalescence process on account of their 
separation exceeding $h_{0}$. The probability density function $p(x, y, \tau)$ which specifies the probability of finding the particle in a differential area around the point $(x, y)$ at time $\tau$ is described by the following Fokker-Planck equation:

$$
\frac{\partial p}{\partial \tau}=-\frac{\alpha \bar{F} T}{\ln \left(h_{c} / h_{0}\right)}(1+y) \frac{\partial p}{\partial x}+\frac{\partial}{\partial y}(y p)+\frac{\delta^{2}}{2 \bar{F}^{2}} \frac{\partial^{2} p}{\partial y^{2}} .
$$

The coalescence efficiency is simply the time integral of the total probability current through the absorbing wall at the left boundary:

$$
\eta=\int_{0}^{x} \mathrm{~d} \tau^{\prime} \int_{-1}^{x} \mathrm{~d} y\left[-\frac{\alpha \bar{F} T}{\ln \left(h_{c} / h_{0}\right)}(1+y) p\left(0, y, \tau^{\prime}\right)\right] .
$$

The coalescence probability can also be estimated from a Monte-Carlo simulation of the system of stochastic differential equations describing the evolution of the force and the film thickness. A large number of sample pathways are constructed following the $O\left(h^{2}\right)$ algorithm for simulating Ito equations derived by Rao et al (1974) and coalescence efficiency is estimated as the fraction of realizations resulting in the abstract particle (describing random motion in a two-dimensional phase plane in accordance with the differential equations) striking the absorbing barrier at $h_{c}$ before hitting $h_{0}$. Muralidhar et al (1988) have obtained excellent agreement between the results of the simulation and an approximate construction using orthonormal polynomials. It is of interest to note the band-limited noise model predicts trends quite opposite to those of the white-noise model. The authors speculate that the neglect of dynamic drop deformation is probably responsible for this difference. They also point out that the general framework for analysis of the coalescence problem as outlined above embeds the random contact time model of Coulaloglou \& Tavlarides (1977).

2.4c Comments on.the models of coalescence frequency: One of the major shortcomings of the coalescence efficiency models is that they do not provide any guidelines for selection of either $h_{0}$ or $h_{c}$. The stability of thin films has yet to be incorporated into the theory and until this is done $h_{c}$ remains an adjustable parameter whose value is chosen to provide the best fit to the experimental data. Furthermore, the models proposed do not permit easy generalization to more complex situations as, for instance, the one wherein surfactants or electrolytes are present in the system. The experimental information on both collision frequency and coalescence efficiency is hard to collect and hence its a vailability is extremely limited. It is difficult to compare various models. The existing models of various complexity can at best be used for first approximation calculations. There is great need for improving the existing models by incorporating the details for calculating $h_{c}$, which has upto this time been taken in an ad hoc manner. When the external phase is rheologically complex, its drainage behaviour will be strongly influenced by its rheological parameters. Some information in this area has recently become available (Hartland \& Jeelani 1987; Bousfield 1989). Similar is the situation for the stability of the thin film intervening between the coalescing drops. Though the basic notion of many tiny fluctuations acting on drops and causing either coalescence or separation is reasonable, the details need deeper analysis. Further, in the wall region of the stirred vessel, where coalescence is predominant, the collisions may occur due to shear rather than the way they have been assumed to occur so far. 
Thus the present state of understanding of both collision frequency and coalescence efficiency is incomplete. There is need for both experimental and theoretical work in this area.

\section{Conclusions}

It is seen that first generation models for both breakage and coalescence of drops in stirred vessels are now available. Though breakage models have been tested against experimental results for dispersed phases of low viscosity, they have hardly been verified for highly viscous liquids and for liquids displaying more complex rheological behaviour. The breakage frequency models for viscous and rheologically complex fluids have not been tested at all. The situation with regard to coalescence frequency is no different. There is strong need both for generating more robust models and for collecting reliable experimental data for verifying them.

\section{List of symbols}

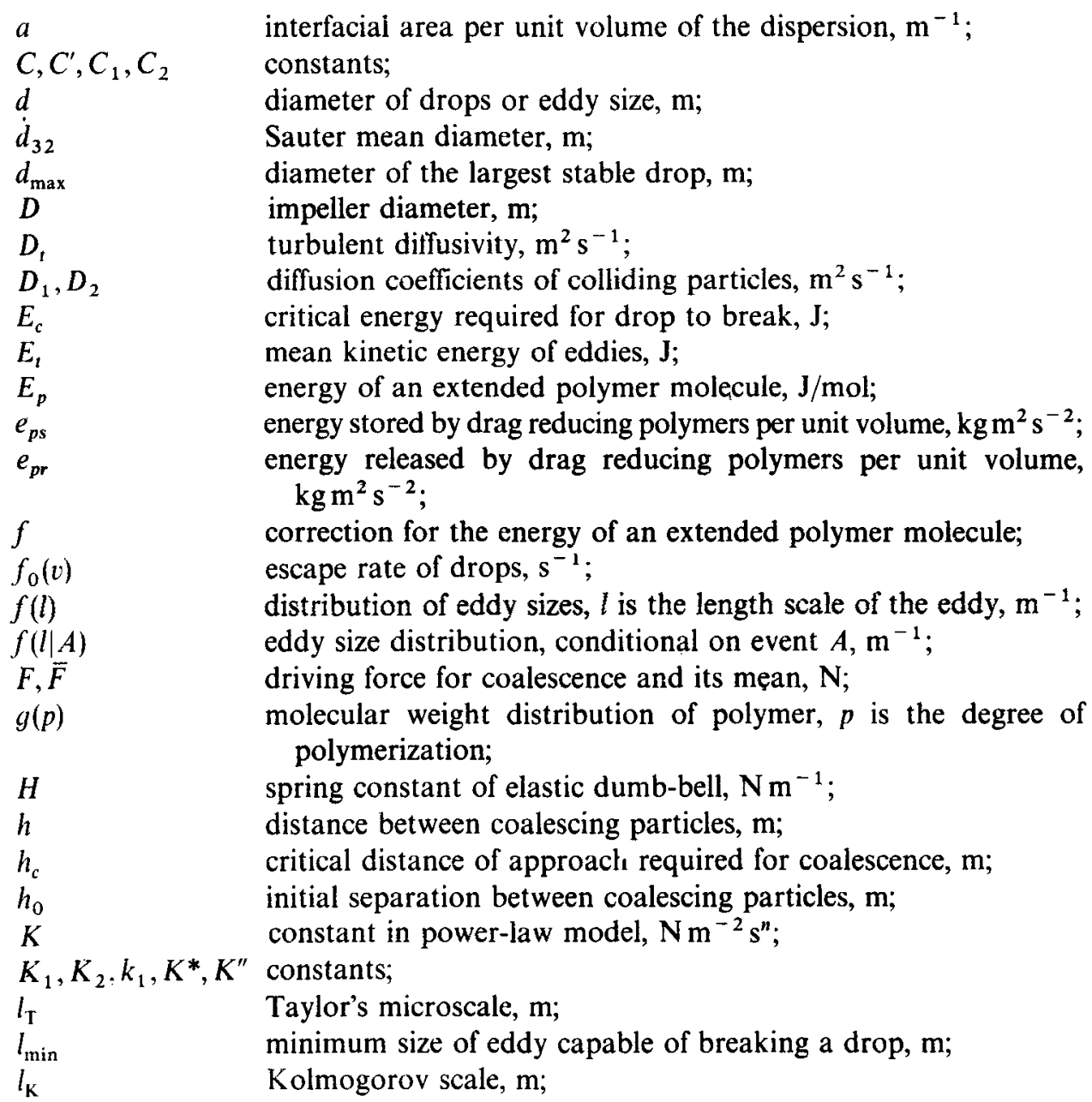




$m_{0}$
$n$
$n(v, t) \mathrm{d} v$
$N$
$N_{0}(v) \mathrm{d} v$
$p$
$q\left(v, v^{\prime}\right)$
$\operatorname{Re}$
$R_{0}$
$R_{g}$
$r_{1}, r_{2}$
$s$
$T$

$T_{l}, T_{d}, T_{f}, T_{t}, T_{s}$ $\bar{T}(l)$

$t_{e x}$

$\frac{t_{b}(l, d)}{u^{2}(d)}$

$t, t^{\prime}$

We

$W_{t}$

$W^{*}$

$w$

$x$

$\beta\left(v, v^{\prime}\right) \mathrm{d} t^{\prime}$

$\delta$

$\varepsilon$

$\eta$

$\phi$

$\because(t)$

$\Gamma(v)$

$\theta$

i.

$\mu$

$v^{\prime}$

'ic

$I^{\prime}\left(t, c^{\prime}\right)$

$\rho$

$\sigma$

$\Delta \sigma$

molecular weight of monomer unit;

power law index:

number of droplets of the dispersed phase in the size range $(v, v+\mathrm{d} v)$

at time $t$ in a unit volume of the dispersion, $\mathrm{m}^{-3}$;

revolutions per second of the stirrer, $s^{-1}$;

number feed rate of drops in the size range $(v, v+\mathrm{d} v)$ per unit volume of the dispersion, $\mathrm{m}^{-3} \mathrm{~s}^{-1}$;

transitional probability density;

frequency of coalescence between drops of size $v$ and $v^{\prime}, \mathrm{m}^{3} \mathrm{~s}^{-1}$; Reynolds number, $D^{n}(N D)^{2-n} \rho_{c} /\left[K 2^{n-3}(3+1 / n)^{n}\right]$;

extended length of the polymer molecule, $\mathrm{m}$;

gas constant, $\mathrm{J} /(\mathrm{mol} \mathrm{K})$;

radii of colliding particles, $\mathrm{m}$;

distance between the centres of particles in collision, $\mathrm{m}$;

temperature, $\mathrm{K}$, and also autocorrelation time of the random force fluctuations, s:

time scales characteristic of coalescence, $s$;

mean lifetime of an eddy of size $l, \mathrm{~s}$;

expected survival time of an eddy, s;

time taken by an eddy of size $l$ to break a drop of diameter $d$, s; mean square velocity fluctuation across a length scale $d, \mathrm{~m}^{2} \mathrm{~s}^{-2}$; drop volume, $\mathrm{m}^{3}$;

Weber number, $\rho_{c} N^{2} D^{3} / \sigma$;

Wiener process;

critical value of kinetic energy required for coalescence, $\mathrm{J}$;

weight of polymer per unit volume, $\mathrm{kg} \mathrm{m}^{-3}$;

defined in (28);

fraction of daughter droplets in the size range $(v, v+\mathrm{d} v)$, when a drop of size $v^{\prime}$ breaks;

standard deviation of stochastic driving force for coalescence;

power dissipation per unit mass, $\mathrm{m}^{2} \mathrm{~s}^{-3}$;

dimensionless time, and also coalescence efficiency;

volume fraction of the dispersed phase;

number of daughter droplets formed on breakage;

breakage frequency, $s^{-1}$;

dimensionless deformation, subscripts $v$ and $s$ refer to deformation due to viscous and interfacial stresses respectively;

relaxation time of polymer, $s$, and also arrival rate of Poisson process, $\mathrm{s}^{-1}$

viscosity, Pa s, subscripts $d$ and $o$ refer to Newtonian and Bingham plastic dispersed phases respectively while $c$ refers to the continuous phase;

frequency of collisions per unit volume, $\mathrm{m}^{-3} \mathrm{~s}^{-1}$;

frequency of collisions, $s^{-1}$;

rate kernel for collisions between drops of size $v$ and $v^{\prime}, \mathrm{m}^{3} \mathrm{~s}^{-1}$; density, subscripts $c$ and $d$ refer to the continuous and dispersed phase, $\mathrm{kg} \mathrm{m}^{-3}$;

interfacial tension. $\mathrm{N} / \mathrm{m}$;

difference between dynamic and static interfacial tensions; 


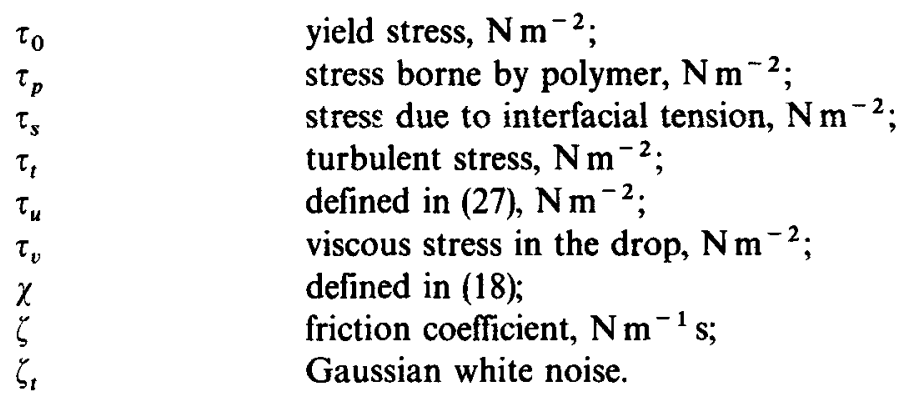

\section{References}

Abrahamson J 1975 Collision rates of small particles in a vigorously turbulent fluid. Chem. Eng. Sci. 30: $1371-1379$

Arai K, Konno M, Matunga Y, Saito S 1977 Effect of dispersed phase viscosity on the maximum stable drop size for break-up in turbulent flow. J. Chem. Eng. Jpn. 10: 325-330

Batchelor G K 1953 Theory of homogeneous turbulence (London: Cambridge University Press)

Benyad S, David R, Cognet G 1985 Measurements of coupled velocity and concentration fluctuations in the discharge flow of a Rushton turbine in a stirred tank. Chem. Eng. Process 19: 157-165

Bousfield D W 1989 Thinning of a viscoelastic film. Chem. Eng. Sci. 44: 763-767

Brodkey R S 1967 The phenomena of fluid motions (Reading: Addison-Wesley)

Bujalski W, Nienow A W, Chatwin S, Cooke M 1987 The dependency on scale of power numbers of Rushton disc turbines. Chem Eng. Sci. 42: 317-326

Calabrese R V, Chang T P K, Dang P T 1986 Drop breakup in turbulent stirred-tank contactors. AIChE J. 32: 657-666

Chappelear D C 1961 Models of a liquid drop approaching an interface. J. Coll. Sci. 16: 186-190

Costes J, Coudrec J P 1988a Study by laser Doppler anemometry of the turbulent flow induced by a Rushton turbine in a stirred tank: influence of the size of the units - I. Mean flow and turbulence. Chem. Eng. Sci. 43: 2751-2764

Costes J, Coudrec J P 1988b Study by laser Doppler anemometry of the turbulent flow induced by a Rushton turbine in a stirred tank: influence of the size of the units - II. Spectral analysis and scales of turbulence. Chem. Eng. Sci. 43: 2765-2772

Coulaloglou C A, Tavlarides L L 1976 Drop size distributions and coalescence frequencies of liquid-liquid dispersions in flow vessels. AIChE J. 22: 289-297

Coulaloglou C A, Tavlarides L L 1977 Description of interaction processes in agitated liquid-liquid dispersions. Chem. Eng. Sci. 32: 1289-1297

Das P K 1984 Analysis of breakage and coalescence of drops in agitated liquid-liquid dispersions, PhD thesis, Indian Inst. Sci., Bangalore

Das P K, Kumar R, Ramkrishna D 1987 Coalescence of drops in stirred dispersion - A white noise model for coalescence. Chem. Eng. Sci. 42: 213-220

Davies J T 1987 Drop sizes of emulsions related to turbulent energy dissipation rates. Chem. Eng. Sci. 42 : $1671-1676$

Harper W R 1936 An exact theory of the coagulation of spherical particles arising from thermal agitation. Trans. Faraday Soc. 32: 1139-1144

Hartland S, Jeelani S A K 1987 Drainage in thin planar non-Newtonian liquid films. Can. J. Chem. Eng. 65: $382-390$

Hinze J O 1955 Fundamentals of the hydrodynamic mechanism of splitting in dispersion processes. AIChE J 289-295

Hoecker H, Langer G, Werner U 1980 Power consumption of stirrers in non-Newtonian liquids. Chemie-Ingr-Tech. 52: 916-917

Howarth W J 1964 Coalescence of drops in a turbulent flow field. Chem. Eng. Sci. 19: 33-38

Hulburt H M, Katz S L 1964 Some problems in particulate technology. Chem. Eng. Sci. 19: 555-574

Kolmogorov A N 1949 On the atomization of droplets in a turbulent flow. Doklady Akad. Nauk. SSSR 66: $825-828$ 
Konno M, Arai K, Saito S 1982 The effect of stabilizer on coalescence of drops in suspension polymerization of styrene. J. Chem. Eng. Jpn 15: 131-135

Koshy A, Das T R, Kumar R 1988a Effect of surfactants on drop breakage in turbulent liquid dispersions. Chem. Eng. Sci. 43: 649 654

Koshy A, Das T R, Kumar R, Gandhi K S 1988b Breakage of viscoelastic drops in turbulent stirred dispersions. Chem. Eng. Sci. 43: 2625-2631

Koshy A, Kumar R, Gandhi K S 1989 Effect of drag reducing agents on drop breakage in stirred dispersions. Chem. Eng. Sci. 44: 2113-2120

Kuboi R, Komasawa I, Otake T 1974 Fluid and particle motion in turbulent dispersions - II. Influence of turbulence of liquid on the motion of suspended particles. Chem. Eng. Sci. 29: 651-658

Laats M K, Frishman F A 1974 Development of technique and investigation of turbulent energy at the axis of a two-phase turbulent jet. Fluid Dyn. 8: 304-307 (transl. from Russian)

Lagisetty J S, Das P K, Kumar R, Gandhi K S 1986 Breakage of viscous and non-Newtonian drops in stirred dispersions. Chem. Eng. Sci. 41: 65-72

Mashelkar R A, Kale D D, Ulbrecht J J 1975 Rotational fows with non-Newtonian fluids - Part 2: torque suppression with agitators. Trans. Inst. Chem. Engrs. 53: 150-153

Muralidhar R, Ramkrishna D 1986 Analysis of droplet coalescence in turbulent liquid-liquid dispersions. I \& EC Fundls. 25: 554-560

Muralidhar R, Ramkrishna D, Das P K, Kumar R 1988 Coalescence of rigid droplets in a stirred dispersion - II. Band-limited force fluctuations. Chem. Eng. Sci. 43: 1559-1568

Narsimhan G, Gupta J P, Ramkrishna D 1979 A model for transitional breakage probability of droplets in agitated lean liquid-liquid dispersions. Chem. Eng. Sci. 34: 257-265

Narsimhan G, Nejfelt G, Ramkrishna D 1984 Breakage functions for droplets in agitated liquid-liquid dispersions. AIChE J. 30: 457-467

Narsimhan G, Ramkrishna D, Gupta J P 1980 Analysis of drop size distributions in lean liquid-liquid dispersions. AIChE J. 26: $991-1000$

Nishikawa M, Okamoto Y, Hashimoto K, Nagata S 1976 Turbulence energy spectra in baffled mixing vessels. J. Chem. Eng. Jpn 9: 489-494

Okamoto Y, Nishikawa M, Hashimoto K 1981 Energy dissipation rate distribution in mixing vessels and its effect on liquid--liquid dispersion and solid-liquid mass transfer. Int. Chem. Eng. 21: 88-94

Papoulis A 1984 Probability, random variables and stochastic processes (New York: McGraw Hill)

Quraishi A Q, Mashelkar R A, Ulbrecht J J 1976 Torque suppression in mechanically stirred liquids and multiphase systems. J. Non-Newtonian Fluid Mech. 1: 223-245

Rallison J M 1984 The deformation of small viscous drops and bubbles in shear flow. Annu. Rev. Fluid Mech. 16: $45-66$

Rao N J, Borwankar J D, Ramkrishna D 1974 Numerical solution of Ito integral equations. SIAM J. Control 12: 124-139

Rietema I 1964 Segregation in liquid-liquid dispersions and its effect on chemical reactions. Adv. Chem. Eny. 5: $237-302$

Ross S L, Curl R L 1973 Measurement and models of the dispersed phase mixing process. Paper 29b, 4th Joint Chem. Eng. Conf., Vancouver, Sept 9-12

Schumpe A, Deckwer D W 1980 Analysis of chemical methods for determination of interfacial areas in gas-in-liquid dispersions with non-uniform bubble sizes. Chem. Eng. Sci. 35: 2221-2233

Shiloh K, Sideman S, Resnick W 1973 Cos lescence and breakage in dilute polydispersions. Can. J. Chem. Eng. 51: 542-549

Smoluchowski M 1917 Versuch einer Mathematischen Theorie der Koagulationskinetik Kolloider Lösungen. Z. Phys. Chem. 92: 129-168

Sprow F B 1967 Distribution of drop sizes produced in turbulent liquid-liquid dispersions. Chem. Eng. Sci. 22: $435-442$

Taylor G I 1921 Diffusion by continuous movements. Proc. London Math. Soc. 20: 196-212

Valentas K J, Amundson N R 1968 Influence of drop size-age distribution on rate processes in dispersed phase systems. I \& EC Fundls. 7: 66-72

Walstra P 1974 Turbulence depression by polymers and its effect on disruption of emulsion droplets. Chem. Eng. Sci. 29: 882-885 